\title{
Undecidability of Propositional Separation Logic and Its Neighbours
}

\author{
JAMES BROTHERSTON, University College London \\ MAX KANOVICH, University College London and Queen Mary, University of London
}

In this article, we investigate the logical structure of memory models of theoretical and practical interest. Our main interest is in "the logic behind a fixed memory model", rather than in "a model of any kind behind a given logical system". As an effective language for reasoning about such memory models, we use the formalism of separation logic. Our main result is that for any concrete choice of heap-like memory model, validity in that model is undecidable even for purely propositional formulas in this language.

The main novelty of our approach to the problem is that we focus on validity in specific, concrete memory models, as opposed to validity in general classes of models.

Besides its intrinsic technical interest, this result also provides new insights into the nature of their decidable fragments. In particular, we show that, in order to obtain such decidable fragments, either the formula language must be severely restricted or the valuations of propositional variables must be constrained.

In addition, we show that a number of propositional systems that approximate separation logic are undecidable as well. In particular, this resolves the open problems of decidability for Boolean BI and Classical BI.

Moreover, we provide one of the simplest undecidable propositional systems currently known in the literature, called "Minimal Boolean BI", by combining the purely positive implication-conjunction fragment of Boolean logic with the laws of multiplicative *-conjunction, its unit and its adjoint implication, originally provided by intuitionistic multiplicative linear logic. Each of these two components is individually decidable: the implication-conjunction fragment of Boolean logic is co-NP-complete, and intuitionistic multiplicative linear logic is NP-complete.

All of our undecidability results are obtained by means of a direct encoding of Minsky machines.

Categories and Subject Descriptors: F.3.1 [Logics and Meanings of Programs]: Specifying and Verifying and Reasoning about Programs_Logics of programs; assertions; F.4.1 [Mathematical Logic and Formal Languages]: Mathematical Logic-Model theory; proof theory

General Terms: Theory, Verification

Additional Key Words and Phrases: Separation logic, undecidability, memory models, bunched logic

ACM Reference Format:

James Brotherston and Max Kanovich. 2014. Undecidability of propositional separation logic and its neighbours. J. ACM 61, 2, Article 14 (April 2014), 43 pages.

DOI: http://dx.doi.org/10.1145/2542667

\section{INTRODUCTION}

In this article, we investigate the logical structure of natural memory models of theoretical and practical interest, our main focus being "the logic behind a fixed memory model" rather than "a model of any kind behind a given logical system". We use separation logic to provide a formula language for such memory models, justified by the fact that, among other things, within this language we can explicitly express basic operations over memories, such as the conflict-free union of disjoint pieces of memory. Furthermore, in the last decade separation logic has become well-established as

J. Brotherston and M. Kanovich are supported by EPSRC.

Authors' address: University College London, Gower Street, London WC1E 6BT, United Kingdom; email: \{j.brotherston, m.kanovich\}@ucl.ac.uk.

Permission to make digital or hard copies of part or all of this work for personal or classroom use is granted without fee provided that copies are not made or distributed for profit or commercial advantage and that copies bear this notice and the full citation on the first page. Copyrights for third-party components of this work must be honored. For all other uses, contact the Owner/Author.

2014 Copyright is held by the author/owner(s)

0004-5411/2014/04-ART14

DOI: http://dx.doi.org/10.1145/2542667 
a practically effective formalism for specifying the properties of such memory models and reasoning about programs that manipulate memory, in the form of pointers, heaps, stacks, etc. [Reynolds 2002; Ishtiaq and O'Hearn 2001; Calcagno et al. 2007]. Automated shape analysis tools based upon separation logic are capable of verifying properties of large industrial programs [Yang et al. 2008; Calcagno et al. 2011], and have been adapted to a variety of paradigms such as object-oriented programming [Parkinson and Bierman 2008; Distefano and Parkinson 2008; Gardner et al. 2012] and concurrent programming [Dodds et al. 2009; Gotsman et al. 2009].

Separation logic is usually based on a memory-oriented mathematical model of heap partitioning. In addition to the standard "additive" Boolean connectives, which are read in the usual way, separation logic features certain "multiplicative" connectives which are interpreted as operations in this model. The most important of these is the so-called separating conjunction $*$, which generally denotes a partial operator for composing heaps whose domains are disjoint: $A_{1} * A_{2}$ denotes the set of heaps which can be split into two disjoint heaps satisfying, respectively, $A_{1}$ and $A_{2}$. The separating conjunction $*$ comes along with its unit I, which denotes the empty heap, and its adjoint implication $A_{1} * A_{2}$, denoting those heaps whose extension with any heap satisfying $A_{1}$, satisfies $A_{2}$. Depending on the application, first-order quantifiers and inductively defined predicates may also be included in the logic.

One of our main contributions in this article is that, irrespective of the choice of underlying memory model, separation logic is undecidable, even at the purely propositional level.

The novelty of our approach to the problem is that we focus on concrete memory models of practical interest, and prove that, whichever concrete heap-like memory model we choose, the validity of propositional separation logic formulas in that model is undecidable. The models we consider-which include the most common memory models used in practice-are listed in Section 2.

As an immediate corollary of this strict result about validity in fixed models, we also obtain undecidability of validity in various classes of such models, and undecidability of provability in several closely related propositional systems.

\subsection{Validity in Fixed Models vs. Validity in Classes of Models}

Validity in a fixed model of practical interest is a much more subtle problem than validity in general classes of models.

As usual, to demonstrate that a validity problem in a classes of models is undecidable, we aim to exhibit a reduction from the halting problem for a suitable class of machines. In our case, these are nondeterministic, 2-counter Minsky machines. Thus, given a formal system, we can construct a provable formula $\mathcal{F}_{M, C}$ in its language which is intended to represent the termination of the Minsky machine $M$ from the configuration $C$. By soundness of the system, provability of $\mathcal{F}_{M, C}$ implies its validity in some class of models.

In order to prove the faithfulness of our encoding, we have to show the converse direction that if $\mathcal{F}_{M, C}$ is valid in this class of models then $M$ terminates from $C$. Now, traditionally, to show that a formula $\mathcal{F}$ has the desired property $Q$ given that $\mathcal{F}$ is valid in a class of models, one constructs some "canonical" model $\mathcal{M}$ in this class such that validity of $\mathcal{F}$ in this specially designed model $\mathcal{M}$ implies $Q$.

However, when we consider the problem of validity in a concrete model $\mathcal{M}_{0}$ specified in advance, we have no such freedom. Instead, we have to show $Q$ given only that $\mathcal{F}$ is valid in this fixed concrete model $\mathcal{M}_{0}$. The difficulty of this task is that there is no ad hoc connection between the given model $\mathcal{M}_{0}$ (of practical interest) and the artificially designed "canonical" model $\mathcal{M}$ one would normally seek to create. 
Since existing decidable fragments of separation logic are based on concrete models, an additional advantage of our approach is that our undecidability results for these models illuminate the restrictions on these fragments. In particular, it transpires that undecidability is closely connected to the tradeoff between finite and infinite valuations of the atomic propositions (cf. Section 10).

Seen as a proof system, separation logic invokes a first-order extension of the propositional bunched logic Boolean BI [Ishtiaq and O'Hearn 2001]. Bunched logics, originating in the "logic of bunched implications" BI [O'Hearn and Pym 1999], can be conceived of as substructural logics that combine a standard propositional logic with various "multiplicative" connectives originally provided by linear logic (cf. Pym [2002] and Brotherston [2012]). Their practical significance for computer science stems from their Kripke-style truth interpretation in which the "worlds" of the Kripke models are understood as resources [Ishtiaq and O'Hearn 2001; Pym et al. 2004; Brotherston and Calcagno 2010].

In proving our main undecidability results, we also establish the undecidability of provability in several propositional systems based on bunched logic. In particular, these include the bunched logics Boolean BI [Ishtiaq and O'Hearn 2001] and Classical BI [Brotherston and Calcagno 2010].

\subsection{Summary of the Structure of the Article}

The remainder of this article is structured as follows:

First, in Section 2, we list a series of natural memory models of theoretical and/or practical interest in various subfields of computer science and mathematics. Then, in Section 3, we give the formula language and semantics of propositional separation logic, which is suitable for expressing properties of these memory models, and, in Section 4, we present a number of propositional logical systems that arise naturally in developing towards an axiomatisation of separation logic and memory models.

An overview of our undecidability results and the proof methodology used to establish them is then given in Section 5. The proofs themselves occupy Sections 6-9, which are mainly technical.

In Section 6, we give a minimal version of Boolean BI, called Minimal BBI, in which negation and falsum are disallowed; this system looks "as simple as possible", but is nonetheless proved to be undecidable.

In Section 7, we encode two-counter Minsky machines as sequents of Minimal BBI so that whenever the machine $M$ terminates from configuration $C$, the corresponding sequent $\mathcal{F}_{M, C}$ is provable in Minimal BBI. The proof of this fact is given in Section 8. By soundness, the sequent $\mathcal{F}_{M, C}$ is then valid in any memory model chosen from Section 2 .

Then, in Section 9 , we show that whenever $\mathcal{F}_{M, C}$ is valid in one of these concrete models listed in Section 2, the machine $M$ must terminate from configuration $C$. The proof of this fact is divided into two stages. First, we consider an intermediate partial $R A M$-domain model (cf. Section 2.5), a degenerate heap model in which the information content of the memory cells is removed, leaving only their "address space". Then, we establish the necessary connection between validity in the RAM-domain model and validity in any of the actual heap-like memory models.

It follows from the main results outlined previously that "any property between provability in Minimal BBI and validity in a heap-like model is undecidable" (see Figure 1, Section 5).

In Section 10, we examine the limitations on decidable fragments of memory models imposed by our undecidability results. Of course, one option for obtaining such decidable fragments is to constrain the formula language so that sequents of the form $\mathcal{F}_{M, C}$ cannot be expressed, which can be done, for example, by excluding the 
multiplicative implication $*$, as happens in Berdine et al. [2004] and in most of the verification tools employing separation logic (cf. Yang et al. [2008], Distefano and Parkinson [2008], and Calcagno et al. [2011]).

Oddly enough, however, it also happens that validity under all finite valuations for atomic propositions in a heap-like model does not imply general validity in that model. Consequently, at least in some models, decidable fragments of memory models can be obtained by restricting valuations to be finite (cf. Calcagno et al. [2001]).

In Section 11, we consider "dualising" separation models and related propositional systems based on Classical BI [Brotherston and Calcagno 2010]. Models of Classical BI differ from the models in Section 2 in that every element is required to have a unique dual; thus, for example, every Abelian group is a model of Classical BI (in which the dual of element is its group inverse), as are bit arrays (where the dual of an array is given by inverting each of its bits). Classical BI is a nonconservative extension of Boolean BI, and thus validity in its models behaves quite differently. Nevertheless, we show that our encoding of Minsky machines also yields undecidability of systems based on Classical BI, and undecidability of validity in associated classes of models.

\subsection{Related Work}

The present article is an extended and revised version of a conference paper which appeared at LICS 2010 [Brotherston and Kanovich 2010].

Here, we report on the main developments in the literature related to our undecidability results, both before and since their original publication in Brotherston and Kanovich [2010].

- The decidability of the bunched logic Boolean BI (BBI, for short) was widely considered an open problem for quite a long time (see, e.g., Galmiche and Larchey-Wendling [2006]). As mentioned previously, undecidability of Boolean BI is a consequence of our general undecidability results for fixed heap-like memory models. However, immediately prior to publication of Brotherston and Kanovich [2010], we discovered that undecidability of BBI can in fact be deduced from the undecidability of equational theories over commutative residual monoids claimed in Kurucz et al. [1995]. More precisely, Kurucz et al. [1995] contains the claim that the equational theory of "CRM" (standing for "Commutative Residuated Monoids"), a certain class of algebras defined in Jipsen's Ph.D. dissertation [Jipsen 1992], is undecidable. Following the reference to Jipsen [1992], one can see that the equational theory of CRM corresponds exactly to the usual notion of provability in BBI. The (rather complicated) proof that CRM is indeed undecidable appears, we believe, only in Kurucz's Ph.D. dissertation [Kurucz 1997].

-Another alternative proof of the undecidability of BBI was given independently by Larchey-Wendling and Galmiche [2010], which appeared at the same time as Brotherston and Kanovich [2010]. Their proof works via an embedding of BBI into a certain fragment of intuitionistic linear logic, where validity according to the standard phase semantics of linear logic is interpreted using a trivial closure operator. Then, they show that their considered fragment of linear logic is both undecidable and complete with respect to this trivial phase semantics. Undecidability of their fragment is based upon a specifically chosen total commutative monoid $(\mathbb{N} \times \mathbb{N},+,(0,0))$ and obtained via an encoding of two-counter Minsky machines (their encoding differs from ours).

-More recently, in Larchey-Wendling and Galmiche [2013], it is additionally shown how to adapt the undecidability proof technique of Larchey-Wendling and Galmiche [2010] to the RAM-domain model (cf. Section 2.5) via bisimulation with the model $(\mathbb{N} \times \mathbb{N},+,(0,0))$. 
In summary, our results, both here and in Brotherston and Kanovich [2010], overlap with Kurucz et al. [1995] and Larchey-Wendling and Galmiche [2010] only with respect to the individual result of undecidability of $\mathrm{BBI}$ (which is obtained by rather different techniques in all cases). The recent journal publication [Larchey-Wendling and Galmiche 2013] overlaps a little further in that it establishes undecidability also for validity in the RAM-domain model (which can be seen as a degenerate heap model in which cell contents, permissions, stacks, etc. are disallowed). The recent journal publication [Larchey-Wendling and Galmiche 2013] also provides an alternative way to establish undecidability for validity in the RAM-domain model (which can be seen as a degenerate heap model in which cell contents, permissions, stacks, etc. are disallowed).

There are at least three main areas in which our work is distinguished from the aforementioned work.

(a) First, we establish the undecidability of validity for any choice of fixed memory model drawn from a spectrum of such models found in the literature, as listed in Section 2.

(b) Second, this proof of undecidability follows from a direct encoding of Minsky machines as "sequents" of the language of separation logic. Though the primary rôle of such an encoding is purely technical, our encoding also enjoys a pedagogically instructive modular structure. Each machine instruction is encoded with a formula that clearly explains the instruction's action. The ability to reuse instructions any number of times is provided by a nontrivial proof principle which we identify as restricted $*$-contraction. From the point of view of our memory models, this principle semantically relies upon the fact that the empty heap can be "split" into two empty heaps.

(c) Third, we shed light on the nature of decidable fragments of separation logic; specifically, we show that to obtain decidability either the formula language or the valuations of propositional variables must be constrained.

We remark that separation logic was conceived as a formalism to aid program verification, where typically a fixed memory model is considered. For this reason, we see the undecidability questions about validity in such models as being of primary importance. There is no ad hoc relationship between such problems and undecidability of validity in simpler models, or indeed undecidability of provability in BBI or related systems, which are typically incomplete. ${ }^{1}$ Of course, it is also gratifying to be able to resolve these latter questions as a matter of historical and/or aesthetic interest. For those interested in such matters, our method does yield undecidability of BBI based on validity in arguably its simplest possible natural model: the total commutative monoid $\left(L^{+}, \cup, \emptyset\right)$, where $L^{+}$is the set of finite multisets over a finite set $L$. See Section 9.5 for details.

\section{MEMORY MODELS OF THEORETICAL AND PRACTICAL INTEREST}

In this section, we list a series of models employing a memory-like concept that are encountered in various areas of computer science and mathematics.

\subsection{Heap Models}

A heap model [Ahmed et al. 2003; Ishtiaq and O'Hearn 2001; Reynolds 2002] is given by

$$
(H, \circ,\{e\})
$$

\footnotetext{
${ }^{1}$ However, Brotherston and Villard [2014] have recently showed how to construct certain extensions of BBI based on hybrid logic that are complete for various classes of memory models.
} 
where $H$ is the set of heaps - that is, finite partial functions from an infinite set of locations $L$ into a set of record values $R V$. The domain of a heap $h$ is the finite set of locations on which $h$ is defined.

The operation $\circ$ is the union of heaps when their domains are disjoint, and undefined otherwise:

$$
h_{1} \circ h_{2}= \begin{cases}h_{1} \cup h_{2} & \text { if } h_{1}, h_{2} \text { have disjoint domains } \\ \text { undefined } & \text { otherwise. }\end{cases}
$$

The unit $e$ of $\circ$ is the empty heap - that is, the function with the empty domain.

(We represent the unit with the singleton $\{e\}$ for consistency with later models employing a set of units rather than a single unit.)

\subsection{Stack-and-Heap Models}

Stack-and-heap models, as used to model Java memory [Parkinson et al. 2006], are given by

$$
(S \times H, \circ, E)
$$

where $H$ is a set of heaps as defined in the previous subsection, and $S$ is a set of stacks, which are finite partial functions from variables Var to stack values Val.

The combination operation $\circ$ for stack-heap pairs is defined as follows:

$$
\left\langle s_{1}, h_{1}\right\rangle \circ\left\langle s_{2}, h_{2}\right\rangle= \begin{cases}\left\langle s_{1}, h_{1} \circ h_{2}\right\rangle & \text { if } s_{1}=s_{2} \text { and } h_{1} \circ h_{2} \text { is defined (by Section 2.1) } \\ \text { undefined } & \text { otherwise. }\end{cases}
$$

Here, the set of units $E$ consists of all pairs $\langle s, e\rangle$ where $s \in S$ and $e$ is the empty heap.

\subsection{Heap-with-Permission Models}

In heap or stack-and-heap models, enforcing the nonoverlapping of domains allows us to avoid possible "read-write" memory conflicts when combining heaps. The heapwith-permission models [Bornat et al. 2005] relax this strict condition; they allow overlapping heaps to be combined, providing that their values agree and "permission" for combination is permitted at all overlapping locations. Permissions are encapsulated by an underlying permission algebra

$$
(P, \bullet, \mathbb{1}),
$$

which is a set $P$ equipped with a partial, commutative, cancellative, and associative operation $\bullet$, and a distinguished element $\mathbb{1}$ (the "write permission") such that $\mathbb{1} \bullet \pi$ is undefined for all $\pi \in P$.

Given such a permission algebra, a heap-with-permission model is then given by

$$
(H, \circ,\{e\}),
$$

where $H$ is the set of heaps-with-permissions, which are finite partial functions from an infinite set of locations $L$ to a set of pairs from $R V \times P$.

We say that two such heaps $h_{1}$ and $h_{2}$ are compatible at $\ell$ if $h_{1}(\ell)=\left\langle v, \pi_{1}\right\rangle$, $h_{2}(\ell)=\left\langle v, \pi_{2}\right\rangle$ and $\pi_{1} \bullet \pi_{2}$ is defined. If $h_{1}$ and $h_{2}$ are compatible at all $\ell$ from the intersection of their domains, then $h_{1} \circ h_{2}$ is defined as follows:

$$
\left(h_{1} \circ h_{2}\right)(\ell)= \begin{cases}\left\langle v, \pi_{1} \bullet \pi_{2}\right\rangle & \text { if } h_{1}(\ell)=\left\langle v, \pi_{1}\right\rangle \text { and } h_{2}(\ell)=\left\langle v, \pi_{2}\right\rangle \\ h_{1}(\ell) & \text { if } h_{1}(\ell) \text { defined and } h_{2}(\ell) \text { undefined } \\ h_{2}(\ell) & \text { if } h_{1}(\ell) \text { undefined and } h_{2}(\ell) \text { defined } \\ \text { undefined } & \text { otherwise. }\end{cases}
$$

If $h_{1}$ and $h_{2}$ are not compatible at some common $\ell$, then $h_{1} \circ h_{2}$ is undefined. As in these heap models, the unit $e$ is the heap with the empty domain. 


\subsection{Stack-and-Heap-with-Permission Models}

Combining the stack-and-heap models of Section 2.2 with the heap-with-permission models of Section 2.3, we obtain the general class of stack-and-heap-with-permission models (cf. Parkinson et al. [2006]), given by

$$
(S \times H, \circ, E)
$$

where $S$ is a set of stacks as defined in Section 2.2, and $H$ is a set of heaps-withpermissions (with underlying permission algebra) as defined in Section 2.3.

The combination of stack and heap-with-permission pairs is then given by the combination operations in Sections 2.2 and 2.3 in the obvious way:

$$
\left\langle s_{1}, h_{1}\right\rangle \circ\left\langle s_{2}, h_{2}\right\rangle= \begin{cases}\left\langle s_{1}, h_{1} \circ h_{2}\right\rangle & \text { if } s_{1}=s_{2} \text { and } h_{1} \circ h_{2} \text { is defined (by Section 2.3) } \\ \text { undefined } & \text { otherwise. }\end{cases}
$$

Again, the set of units $E$ consists of all pairs $\langle s, e\rangle$ where $s \in S$ and $e$ is the empty heap.

\subsection{The RAM-Domain Model}

In order to simplify our undecidability constructions, we consider a degenerate version of the heap model in which we remove the information contents of cells, retaining only their "address space". This model, that is "as simple as possible but no simpler", is the $R A M$-domain model of Aho et al. [1974], given by

$$
\left(\mathcal{D}, \circ,\left\{e_{0}\right\}\right),
$$

where $\mathcal{D}$ is the class of finite subsets of $\mathbb{N}$, to be understood as finite sets of memory locations. The operation $\circ$ denotes a partial operation for combining disjoint sets of locations:

$$
d_{1} \circ d_{2}= \begin{cases}d_{1} \cup d_{2}, & \text { if } d_{1} \cap d_{2}=\emptyset \\ \text { undefined, } & \text { otherwise }\end{cases}
$$

Here, $e_{0}$ is defined to be the neutral element or unit of $\circ$, that is, the empty set.

Remark 2.1. Any of these models can be seen as a special case of a stack-and-heapwith-permission model from Section 2.4, obtained by an appropriate choice of locations $L$, values $R V$, stacks $S$ and permission algebra $(P, \bullet, \mathbb{1})$.

\subsection{Multiset/Integer Partition Models}

Models related to integer partitions [Andrews 1976] are given by

$$
\left(H, \circ,\left\{e_{0}\right\}\right),
$$

where $H$ is a set of finite integer multisets, with $\circ$ being the total operation of multiset union, and the unit $e_{0}$ being the empty multiset.

\subsection{Petri-Net Models}

Models of Petri-net markings [Peterson 1981; Murata 1989] are given by

$$
\left(H, \circ,\left\{e_{0}\right\}\right),
$$

where for some $K \subseteq \mathbb{N}$ and some set $L$ of locations, the set $H$ is the set of markings, defined as finite partial functions from $L$ into $K$.

By taking $K=\mathbb{N}$, we model states in traditional Petri nets, with $\circ$ being the total operation of multiset union, that is, $\left(h_{1} \circ h_{2}\right)(\ell)=h_{1}(\ell)+h_{2}(\ell)$.

By taking $K=\{0,1, \ldots, k\}$, we model states in Petri nets with capacity $k$, where $\circ$ is multiset union as previously mentioned, but with $\left(h_{1} \circ h_{2}\right)(\ell)$ undefined when $h_{1}(\ell)+h_{2}(\ell)>k$. 
The unit $e_{0}$ is the empty marking, that is, the marking with empty domain.

These models can be also thought of as a certain kind of memory model, where every location $\ell$ has an associated size or "number of blocks" $h(\ell)$.

\section{PROPOSITIONAL SEPARATION LOGIC: A FORMALISM FOR REASONING ABOUT MEMORY MODELS}

Here we present the language of propositional separation logic and its interpretation in the usual class of "separation models" from Calcagno et al. [2007], which abstract from the concrete memory models employed in the literature (cf. Section 2).

Definition 3.1. A separation model is a cancellative partial commutative monoid

$$
(H, \circ, E) \text {, }
$$

where $\circ$ is a partial binary operation on $H$ which is associative and commutative. The equality of expressions $\alpha=\beta$ means that either $\alpha$ and $\beta$ are both undefined, or $\alpha$ and $\beta$ are defined and equal. Cancellativity of $\circ$ means that if $h \circ h^{\prime}$ is defined and $h \circ h^{\prime}=h \circ h^{\prime \prime}$, then $h^{\prime}=h^{\prime \prime}$.

We extend $\circ$ to a multiplication $\cdot$ on subsets of $H$ by

$$
X \cdot Y=_{\text {def }}\left\{h \circ h^{\prime} \mid h \in X, h^{\prime} \in Y \text {, and } h \circ h^{\prime} \text { is defined }\right\} .
$$

The set of units $E$ is then a subset of $H$ such that, for all $h \in H$,

$$
E \cdot\{h\}=\{h\} .
$$

All the models from Section 2 are separation models. We allow a set of units $E$ rather than a single unit $e$ in Definition 3.1 in order to cover the whole spectrum of heap-like models (e.g., the stack-and-heap models in Section 2.2).

Proposition 3.2. For any $h \in H$, there is a unique element $e \in E$ such that $h \circ e=h$.

Proof. The existence of a suitable $e$ is guaranteed by $h \circ E=\{h\}$. For uniqueness, assume $h \circ e=h \circ e^{\prime}=h$. Then, by cancellativity, we get $e=e^{\prime}$.

Proposition 3.3. The set of units $E$ in a separation model $(H, \circ, E)$ forms a "unit matrix". That is, for any $e_{i}, e_{j} \in E$, we have:

$$
e_{i} \circ e_{j}= \begin{cases}e_{i}, & \text { if } e_{i}=e_{j}, \\ \text { undefined, } & \text { otherwise } .\end{cases}
$$

In particular, if $\circ$ is total, then $E$ is forced to be a singleton $\{e\}$.

Proof. Let $e_{i}, e_{j} \in E$. Using (2) and commutativity of $\circ$, we have $\left\{e_{i}\right\} \cdot E=\left\{e_{i}\right\}$ whence $e_{i} \circ e_{j}$ is either $e_{i}$ or undefined. But, by the same token, $E \cdot\left\{e_{j}\right\}=\left\{e_{j}\right\}$ whence $e_{i} \circ e_{j}$ is either $e_{j}$ or undefined. Thus, if $e_{i} \neq e_{j}$, then $e_{i} \circ e_{j}$ must be undefined. To see that $e_{i} \circ e_{i}=e_{i}$, observe that we must have $e_{i} \circ e=e_{i}$ for some $e \in E$ because $e_{i} \in\left\{e_{i}\right\} \cdot E$.

Definition 3.4. A separation model $(H, \circ, E)$ is said to have indivisible units if $h_{1} \circ h_{2} \in E$ implies $h_{1} \in E$ and $h_{2} \in E$ for all $h_{1}, h_{2} \in H$.

(In fact, in this case $h_{1}=h_{2}$ because of the observation in Proposition 3.3 that $h_{1} \circ h_{2}$ must be undefined when $h_{1} \neq h_{2}$.)

Remark 3.5. The memory models employed in the literature (see Section 2 ) all have indivisible units in the sense of Definition 3.4. This natural property can be summarised by the following slogan on "conservation of matter":

“The empty memory cannot be split into non-empty pieces". 
However, in contrast to the memory models, we can easily construct a separation model whose units are divisible; for example, the model $(\mathbb{Z},+,\{0\})$, where + is addition on the integers $\mathbb{Z}$, is one such.

Definition 3.6. Formulas are built from an infinite set of atomic propositions $p$ and constants $T, \perp$, and I, by a unary operator $\neg$ and binary connectives $\wedge, \vee, \rightarrow, *$, and $\rightarrow$. (We call $*$ the multiplicative conjunction, with $*$ its associated adjoint implication and I its unit.)

For the sake of readability, we often write a formula of the form $A \rightarrow B$ as the "sequent" $A \vdash B$.

By convention, negation $\neg$ has the greatest precedence, followed by $\wedge, \vee$, and $*$, with the implications $\rightarrow$ and $*$ having lowest precedence.

Definition 3.7. A valuation for a separation model $(H, \circ, E)$ is a function $\rho$ that assigns to each atomic proposition $p$ a set $\rho(p) \subseteq H$. Given any $h \in H$ and formula $A$, we define the forcing relation $h \models_{\rho} A$ by induction on $A$ :

$$
\begin{aligned}
h \models_{\rho} p & \Leftrightarrow h \in \rho(p) \\
h \models_{\rho} T & \Leftrightarrow \text { always } \\
h \models_{\rho} \perp & \Leftrightarrow \text { never } \\
h \models_{\rho} \neg A & \Leftrightarrow h \models_{\rho} A \\
h \models_{\rho} A_{1} \wedge A_{2} & \Leftrightarrow h=_{\rho} A_{1} \text { and } h=_{\rho} A_{2} \\
h \models_{\rho} A_{1} \vee A_{2} & \Leftrightarrow h=_{\rho} A_{1} \text { or } h \models_{\rho} A_{2} \\
h \models_{\rho} A_{1} \rightarrow A_{2} & \Leftrightarrow \text { if } h \models_{\rho} A_{1} \text { then } h \models_{\rho} A_{2} \\
h \models_{\rho} \mathrm{I} & \Leftrightarrow h \in E \\
h \models_{\rho} A_{1} * A_{2} & \Leftrightarrow \exists h_{1}, h_{2} . h=h_{1} \circ h_{2} \text { and } h_{1} \models_{\rho} A_{1} \text { and } h_{2} \models_{\rho} A_{2} \\
h \models{ }_{\rho} A_{1} * A_{2} & \Leftrightarrow \forall h^{\prime} . \text { if } h \circ h^{\prime} \text { defined and } h^{\prime} \models_{\rho} A_{1} \text { then } h \circ h^{\prime} \models_{\rho} A_{2} .
\end{aligned}
$$

The intended meaning of any formula $A$ under $\rho$ is given by

$$
\llbracket A \rrbracket_{\rho}=_{\text {def }}\left\{h \mid h \models_{\rho} A\right\} .
$$

Lemma 3.8. Given a separation model $(H, \circ, E)$ and a valuation $\rho$ for the model, we have the following identities:

$$
\begin{aligned}
\llbracket p \rrbracket_{\rho} & =\rho(p) \\
\llbracket \perp \rrbracket_{\rho} & =\emptyset \\
\llbracket \mathrm{I} \rrbracket_{\rho} & =E \\
\llbracket A \wedge B \rrbracket_{\rho} & =\llbracket A \rrbracket_{\rho} \cap \llbracket B \rrbracket_{\rho} \\
\llbracket A * B \rrbracket_{\rho} & =\llbracket A \rrbracket_{\rho} \cdot \llbracket B \rrbracket_{\rho} \\
\llbracket A \rightarrow B \rrbracket_{\rho} & =\text { largest } Z \subseteq H . \llbracket A \rrbracket_{\rho} \cap Z \subseteq \llbracket B \rrbracket_{\rho} \\
\llbracket A \rightarrow B \rrbracket_{\rho} & =\text { largest } Z \subseteq H . \llbracket A \rrbracket_{\rho} \cdot Z \subseteq \llbracket B \rrbracket_{\rho} .
\end{aligned}
$$

Proof. We just show the identities (7) and (9). In the case of (7), we have:

$$
\begin{aligned}
\llbracket A * B \rrbracket_{\rho} & =\left\{h \mid \exists h_{1}, h_{2} . h=h_{1} \circ h_{2} \text { and } h_{1} \models_{\rho} A \text { and } h_{2} \models_{\rho} B\right\} \\
& =\left\{h_{1} \circ h_{2} \mid h_{1} \models_{\rho} A \text { and } h_{2} \models_{\rho} B \text { and } h_{1} \circ h_{2} \text { defined }\right\} \\
& =\left\{h_{1} \circ h_{2} \mid h_{1} \in \llbracket A \rrbracket_{\rho} \text { and } h_{2} \in \llbracket B \rrbracket_{\rho} \text { and } h_{1} \circ h_{2} \text { defined }\right\} \\
& =\llbracket A \rrbracket_{\rho} \cdot \llbracket B \rrbracket_{\rho} .
\end{aligned}
$$


To show (9), we first show that $\llbracket A * B \rrbracket_{\rho}$ satisfies the required inclusion, that is

$$
\llbracket A \rrbracket_{\rho} \cdot \llbracket A \rightarrow B \rrbracket_{\rho} \subseteq \llbracket B \rrbracket_{\rho} .
$$

To see this, let $h \in \llbracket A \rrbracket_{\rho} \cdot \llbracket A \rightarrow B \rrbracket_{\rho}$. Then there are $h_{1}, h_{2} \in H$ with $h=h_{1} \circ h_{2}$ and $h_{1} \models{ }_{\rho} A$ and $h_{2} \models{ }_{\rho} A * B$. In particular, this means that for any $h^{\prime}$ such that $h^{\prime} \circ h_{2}$ is defined and $h^{\prime} \models_{\rho} A$, we have $h^{\prime} \circ h_{2} \models_{\rho} B$. Since $h_{1} \circ h_{2}$ is defined, we have $h_{1} \circ h_{2} \models_{\rho} B$, that is, $h \models{ }_{\rho} B$ as required.

To see that $\llbracket A \rightarrow B \rrbracket_{\rho}$ is the largest set satisfying the required inclusion, we let $Z \subseteq H$ satisfy $\llbracket A \rrbracket_{\rho} \cdot Z \subseteq \llbracket B \rrbracket_{\rho}$, and show that $Z \subseteq \llbracket A * B \rrbracket_{\rho}$. This means showing that if $h \in Z$, then $h \models_{\rho} A * B$. Let $h^{\prime} \in H$ be such that $h \circ h^{\prime}$ is defined and $h^{\prime} \models_{\rho} A$, that is, $h^{\prime} \in \llbracket A \rrbracket_{\rho}$. Since $\llbracket A \rrbracket_{\rho} \cdot Z \subseteq \llbracket B \rrbracket_{\rho}$, we have $h \circ h^{\prime} \in \llbracket B \rrbracket_{\rho}$, that is, $h \circ h^{\prime} \models_{\rho} B$ as required.

Definition 3.9. A formula $A$ is valid in a separation model $(H, \circ, E)$ if for any valuation $\rho$, we have $\llbracket A \rrbracket_{\rho}=H$.

In particular, a sequent $A \vdash B$ is valid in $(H, \circ, E)$ if $\llbracket A \rrbracket_{\rho} \subseteq \llbracket B \rrbracket_{\rho}$ for any valuation $\rho$.

In the next theorem, we establish the exact correlation between seemingly different concepts: the indivisibility of units in the memory models, and the restricted *-weakening in formal systems, given by axioms of the form

$$
\mathrm{I} \wedge(A * B) \vdash A \text {. }
$$

THEOREM 3.10 .

(a) For any formulas $A$ and $B$, the restricted $*$-weakening principle

$$
\mathrm{I} \wedge(A * B) \vdash A
$$

is valid in all separation models $(H, \circ, E)$ with indivisible units.

(b) In addition, the above correlation is exact. Namely, the basic instance of the restricted *-weakening principle (here $p$ and $q$ are atomic propositions),

$$
\mathrm{I} \wedge(p * q) \vdash p
$$

is valid in a separation model $(H, \circ, E)$ if and only if $(H, \circ, E)$ has indivisible units.

Proof.

(a) Let $(H, \circ, E)$ be a separation model with indivisible units, let $h \in H$ and suppose that $h \models_{\rho} \mathrm{I} \wedge(A * B)$. Thus, $h \in E$ and there exist $h_{1}, h_{2} \in H$ with $h=h_{1} \circ h_{2}$ and $h_{1} \models_{\rho} A$ and $h_{2}={ }_{\rho} B$. As $(H, \circ, E)$ has indivisible units, we have $h_{1} \in E$ and $h_{2} \in E$, whence by Proposition 3.3, we have $h_{1}=h_{2}=h$. Thus, $h \models_{\rho} A$ and, as $h$ was arbitrarily chosen, $\mathrm{I} \wedge(A * B) \vdash A$ is valid in this model as required.

(b) Let $\left(H^{\prime}, \circ^{\prime}, E\right)$ be a separation model whose set of units $E$ is divisible, that is, such that $h^{\prime} \circ^{\prime} h^{\prime \prime}=e \in E$ for some $h^{\prime} \notin E$.

Let $\rho$ be a valuation with $\rho(p)=\left\{h^{\prime}\right\}$ and $\rho(q)=\left\{h^{\prime \prime}\right\}$. Then, we easily have that $e \models_{\rho} I \wedge(p * q)$ but $e \not \models_{\rho} p$, so $\mathrm{I} \wedge(p * q) \vdash p$ is not valid in this model as required.

The following proposition shows that separation models with total and nontotal compositions $\circ$ behave quite differently with respect to validity.

Proposition 3.11. A sequent of the form

$$
\mathrm{I} \wedge(p * q * \perp) \vdash(p * \perp) \vee(q * \perp)
$$

is valid in a separation model $(H, \circ, E)$ if and only if the operation $\circ$ is total.

PRoof. In fact, the sequent (10) represents the following "natural" law:

$$
\llbracket p \rrbracket_{\rho} \cdot \llbracket q \rrbracket_{\rho}=\emptyset \text { implies }\left(\llbracket p \rrbracket_{\rho}=\emptyset \text { or } \llbracket q \rrbracket_{\rho}=\emptyset\right) \text {. }
$$


$(\Leftarrow)$ Let $(H, \circ, E)$ be a separation model with $\circ$ total, and suppose $h \models_{\rho} \mathrm{I} \wedge(p * q \rightarrow \perp)$. Then, $h \in E$ and $h=_{\rho} p * q * \perp$. Using Lemma 3.8, we have $\llbracket p * q \rrbracket_{\rho} \subseteq \llbracket \perp \rrbracket_{\rho}=\emptyset$, and thus $\llbracket p \rrbracket_{\rho}: \llbracket q \rrbracket_{\rho}=\llbracket p * q \rrbracket_{\rho}=\emptyset$.

Since $\circ$ is total, $\llbracket p \rrbracket_{\rho} \cdot \llbracket q \rrbracket_{\rho}=\emptyset$ implies that either $\llbracket p \rrbracket_{\rho}=\emptyset$ or $\llbracket q \rrbracket_{\rho}=\emptyset$. Consequently, either $h \models_{\rho} p * \perp$ or $h \models_{\rho} q * \perp$, i.e. $h=_{\rho}(p * \perp) \vee(q * \perp)$ as required.

$(\Rightarrow)$ Let $(H, \circ, E)$ be a separation model in which $\circ$ is nontotal, that is for some $h^{\prime}$ and $h^{\prime \prime}$, their product $h^{\prime} \circ h^{\prime \prime}$ is undefined. Let $\rho$ be a valuation with $\rho(p)=\left\{h^{\prime}\right\}$ and $\rho(q)=\left\{h^{\prime \prime}\right\}$. Then, $\llbracket p \rrbracket_{\rho} \cdot \llbracket q \rrbracket_{\rho}=\emptyset$ by construction. Thus, using Lemma 3.8, we have

$$
\begin{aligned}
E \cdot \llbracket p * q \rrbracket_{\rho} & =\llbracket p * q \rrbracket_{\rho} \\
& =\llbracket p \rrbracket_{\rho} \cdot \llbracket q \rrbracket_{\rho} \\
& =\emptyset \\
& =\llbracket \perp \rrbracket_{\rho} .
\end{aligned}
$$

Thus, in particular, $E \cdot \llbracket p * q \rrbracket_{\rho} \subseteq \llbracket \perp \rrbracket_{\rho}$ and, by Lemma 3.8, we have $E \subseteq \llbracket p * q * \perp \rrbracket_{\rho}$, which means that $\llbracket \mathrm{I} \wedge(p * q * \perp) \rrbracket_{\rho} \neq \emptyset$. On the other hand, by construction, both $\llbracket p * \perp \rrbracket_{\rho}=\emptyset$ and $\llbracket q * \perp \rrbracket_{\rho}=\emptyset$, that is, $\llbracket(p * \perp) \vee(q * \perp) \rrbracket_{\rho}=\emptyset$. We conclude that our sequent is not valid in this model as required.

\section{FORMAL SYSTEMS FOR SEPARATION LOGIC}

In this section, we explore various notions of provability in the language of propositional separation logic, and examine their connection to validity in various classes of models. First, in Section 4.1, we introduce the core axiomatisation of the multiplicative connectives $*, *$ and I. Then, in Section 4.2, we examine a chain of logical systems extending this axiomatisation, including the well-known bunched logics BI and Boolean BI.

\subsection{Axiomatisation of the Multiplicatives}

The mathematical behaviour of separation models $(H, \circ, E)$ (see Definition 3.1), and the interpretation of the multiplicative connectives $*, *$ and I (Definition 3.7) naturally gives rise to a number of axioms and rules for these connectives, as follows.

(a) First, the combination operation $\circ$ is associative and commutative by definition, which means that the multiplicative conjunction $*$ should also be associative and commutative:

$$
A * B \vdash B * A \quad \text { and } \quad A *(B * C) \vdash(A * B) * C .
$$

(b) Next, the set $E$ is a unit for $\circ$, that is, $E \circ\{h\}=\{h\}$, which means that I (representing $E$ ) should also be a unit for $*$ :

$$
A * \mathrm{I} \vdash A \quad \text { and } \quad A \vdash A * \mathrm{I} .
$$

(c) The "magic wand" connective $*$ is defined as the implication adjoint to the conjunction $*$, which leads to the following residuation principles for $*$ and $*$ :

$$
A *(A * B) \vdash B \quad \text { and } \quad \frac{A * B \vdash C}{A \vdash B * C} .
$$

(d) Finally, we have the following monotonicity or "frame" principle for building entailments between $*$-conjunctions:

$$
\frac{A \vdash B}{A * C \vdash B * C} .
$$


In fact, this collection of axioms and inference rules for the multiplicatives is originally provided by intuitionistic multiplicative linear logic, IMLL [Girard and Lafont 1987; Benton et al. 1993].

\subsection{Bunched Logic Proof Systems for Separation Logic}

Core proof systems for propositional separation logic are provided by bunched logic, a class of substructural logics pioneered by O'Hearn and Pym. Specifically, the propositional proof theory of separation logic is usually considered to be given by the bunched logic Boolean BI, although other choices are also possible. Every separation model can be seen as a model of Boolean BI [Ishtiaq and O'Hearn 2001; Galmiche and LarcheyWendling 2006].

Definition 4.1. We consider the following chain of logical systems

$$
\mathrm{BI} \subseteq \mathrm{BBI} \subseteq \mathrm{BBI}+\mathrm{eW} \subseteq \mathrm{BBI}+\mathrm{W},
$$

each defined as follows:

-BI, a.k.a. the logic of bunched implications (cf., O'Hearn and Pym [1999], Pym [2002], and Galmiche et al. [2005]) is given by:

(A) all instances of intuitionistically valid formulas and inference rules, and

(B) the axioms and inference rules for $*, *$ and I given in Section 4.1.

-Boolean BI, a.k.a. BBI (cf., Ishtiaq and O'Hearn [2001] and Galmiche and LarcheyWendling [2006]) is obtained from BI by expanding (A) above to include all instances of classically valid propositional formulas and inference rules.

-As we shall see (Lemma 6.4), the restricted $*$-contraction

$$
\mathrm{I} \wedge A \vdash A * A
$$

holds in BBI, whereas the analogous restricted $*$-weakening

$$
\mathrm{I} \wedge(A * B) \vdash A
$$

does not. However, the restricted $*$-weakening exactly expresses the indivisibility of units in a separation model (see Theorem 3.10). Thus, we introduce the system $\mathrm{BBI}+\mathrm{eW}$ by enriching BBI with all new axioms of the form $\mathrm{I} \wedge(A * B) \vdash A$.

-Having considered restricted $*$-weakening, it is also natural to consider $\mathrm{BBI}+\mathrm{W}$, obtained by enriching BBI with the unrestricted $*$-weakening - that is, axioms of the form

$$
A * B \vdash A
$$

(or, equivalently, $B \vdash \mathrm{I}$ ).

Proposition 4.2 (Soundness of BBI). If $A$ is provable in $\mathrm{BBI}$, then $A$ is valid in all separation models.

Proof. We just need to show that validity in any separation model is preserved by the axioms and rules of classical propositional logic and by the axioms and rules given in Section 4.1, which is an easy exercise.

In many cases, the connection between provability in the systems in Definition 4.1 and validity in the classes of corresponding separation models is not exact. For example, BBI is not complete even for validity in the class of all partial commutative monoids [Larchey-Wendling and Galmiche 2010]. No complete natural axiomatisation 
is currently known for validity with respect to the class of all separation models (or an interesting subclass thereof).

Corollary 4.3 (Soundness of BBI $+\mathrm{eW}$ ). If Ais provable in $\mathrm{BBI}+\mathrm{eW}$, then Ais valid in all separation models with indivisible units.

Proof. Immediate from Proposition 4.2 and Theorem 3.10.

CoROLlary 4.4. Interpreting $\subset$ as strict inclusion between the sets of sequents provable in each system, we have:

$$
\mathrm{BI} \subset \mathrm{BBI} \subset \mathrm{BBI}+\mathrm{eW} \subset \mathrm{BBI}+\mathrm{W} .
$$

Proof. First, note that the non-strict inclusions $\mathrm{BI} \subseteq \mathrm{BBI} \subseteq \mathrm{BBI}+\mathrm{eW} \subseteq \mathrm{BBI}+\mathrm{W}$ hold easily by the construction of the systems in Definition 4.1. Thus, we just need to show $\mathrm{BBI}+\mathrm{W} \nsubseteq \mathrm{BBI}+\mathrm{eW} \nsubseteq \mathrm{BBI} \nsubseteq \mathrm{BI}$.

First, BBI $\nsubseteq$ BI holds because BI is conservative over intuitionistic logic [Pym 2002] and thus, for example, the classical tautology $(p \rightarrow \perp) \rightarrow \perp \vdash p$ is invalid in BI, whereas it is valid in BBI.

$\mathrm{BBI}+\mathrm{eW} \nsubseteq \mathrm{BBI}$ holds because, by Theorem 3.10 , the instance $\mathrm{I} \wedge(p * q) \vdash p$ of $\mathrm{BBI}+\mathrm{eW}$ 's restricted $*$-weakening axiom is not valid in all separation models, and hence not provable in BBI by Proposition 4.2.

Finally, BBI $+\mathrm{W} \nsubseteq \mathrm{BBI}+\mathrm{eW}$ holds because the instance $p \vdash \mathrm{I}$ of $\mathrm{BBI}+\mathrm{W}$ 's unrestricted $*$-weakening axiom is not valid in all separation models with indivisible units, and hence not provable in $\mathrm{BBI}+\mathrm{eW}$ by Corollary 4.3 . To see this, let $(H, \circ, E)$ be a separation model with indivisible units, and let $\rho(p)=\{h\}$ for some $h \notin E$. Then, easily, $h \models \rho p$ but $h \not{ }_{\rho} \mathrm{I}$.

One of the important features of separation logic is that the $*$-contraction, $A \vdash A * A$, is not generally valid, and hence not provable in BBI by Proposition 4.2. Surprisingly, however, BBI does enjoy the restricted $*$-contraction (see Lemma 6.4 in Section 6)

$$
\mathrm{I} \wedge A \vdash A * A,
$$

which holds only at the level of the multiplicative unit I.

Remark 4.5. We note that the following basic instance of restricted $*$-contraction

$$
\mathrm{I} \wedge p \vdash p * p
$$

is not valid in ordinary intuitionistic BI. This can most easily be seen from the elementary Kripke semantics of BI in Galmiche et al. [2005], whereby models are ordered partial commutative monoids $(M, \circ, e, \sqsubseteq)$, and we have the following clauses for satisfaction of formulas by $m \in M$ and valuation $\rho$ :

$$
\begin{aligned}
m \models_{\rho} \mathrm{I} & \Leftrightarrow e \sqsubseteq m \\
m \models_{\rho} A \wedge B & \Leftrightarrow m \models_{\rho} A \text { and } m \models_{\rho} B . \\
m \models{ }_{\rho} A * B & \Leftrightarrow \exists n, n^{\prime} \in M . n \circ n^{\prime} \leq m \text { and } n \models_{\rho} A \text { and } n \models_{\rho} B .
\end{aligned}
$$

Now consider the 2-element BI-model

$$
(\{e, b\}, \circ, e, \sqsubseteq),
$$

where $\circ$ is given by $e \circ e=b \circ b=e$ and $e \circ b=b \circ e=b$, and $\sqsubseteq$ is given by $e \sqsubset b$. Let $\rho$ be a valuation with $\rho(p)=\{b\}$. Then, easily, $b \models_{\rho} \mathrm{I} \wedge p$ but $b \not{ }_{\rho} p * p$, that is, $\mathrm{I} \wedge p \vdash p * p$ is not valid in this model. ${ }^{2}$

\footnotetext{
${ }^{2}$ The key difference compared to validity in separation models is that, in the satisfaction relation for BBI in Definition 3.7, $b \models_{\rho} \mathrm{I} \wedge p$ forces $e=b$, while in the satisfaction relation for BI, it only forces $e \sqsubseteq b$.
} 
In the case of $\mathrm{BBI}+\mathrm{eW}$, its restricted $*$-weakening with the restricted $*$-contraction of Lemma 6.4 induces a collapse of $\wedge$ and $*$ at the level of the unit I, as follows.

Corollary 4.6. The following equivalences hold in $\mathrm{BBI}+\mathrm{eW}$ :

$$
\mathrm{I} \wedge(A * B) \equiv \mathrm{I} \wedge A \wedge B \equiv(\mathrm{I} \wedge A) *(\mathrm{I} \wedge B),
$$

where the equivalence $F \equiv G$ means that both $F \vdash G$ and $G \vdash F$ are provable.

Proof. First, $I \wedge(A * B) \vdash I \wedge A \wedge B$ is derivable using the restricted $*$-weakening. Second, using the restricted $*$-contraction and the usual contraction for $\wedge$ we can derive:

$$
\mathrm{I} \wedge A \wedge B \vdash(\mathrm{I} \wedge A \wedge B) *(\mathrm{I} \wedge A \wedge B),
$$

whence $\mathrm{I} \wedge A \wedge B \vdash(\mathrm{I} \wedge A) *(\mathrm{I} \wedge B)$ follows from weakening for $\wedge$. Finally, we can derive $(\mathrm{I} \wedge A) *(\mathrm{I} \wedge B) \vdash \mathrm{I} \wedge(A * B)$ using the equivalence $\mathrm{I} * C \equiv C$ and weakening for $\wedge$. The required equivalences then follow by transitivity of $\vdash$.

Proposition 4.7. BBI $+\mathrm{W}$ collapses into ordinary classical logic.

Proof. Trivially, $\mathrm{I} \wedge A \vdash A$ is provable in $\mathrm{BBI}+\mathrm{W}$. Using the equivalence $\mathrm{I} * A \equiv A$ and contraction for $\wedge$, we also have $A \vdash(\mathrm{I} * A) \wedge A$ provable. Using the unrestricted *-weakening of $\mathrm{BBI}+\mathrm{W}$, we obtain $A \vdash \mathrm{I} \wedge A$, and thus $\mathrm{I} \wedge A \equiv A$ holds in $\mathrm{BBI}+\mathrm{W}$. Using this equivalence together with Corollary 4.6, we have:

$$
A * B \equiv \mathrm{I} \wedge(A * B) \equiv \mathrm{I} \wedge A \wedge B \equiv A \wedge B
$$

which also guarantees that $A * B \equiv A \rightarrow B$. Finally, using the equivalence $\mathrm{I} \wedge A \equiv A$, we have $\mathrm{I} \equiv \mathrm{I} \wedge \mathrm{T} \equiv \mathrm{T}$. Thus, all the multiplicative connectives of $\mathrm{BBI}+\mathrm{W}$ collapse into their classical additive equivalents.

Remark 4.8. Both ends of the chain of logics $\mathrm{BI} \subset \mathrm{BBI} \subset \mathrm{BBI}+\mathrm{eW} \subset \mathrm{BBI}+\mathrm{W}$ given by Corollary 4.4 are in fact decidable. BI was shown decidable in Galmiche et al. [2005], and BBI $+\mathrm{W}$ is decidable because, by Proposition 4.7, it collapses into ordinary classical logic. Thus, technically speaking, it is relatively surprising that-as we shall see in the next section-the intermediate systems BBI and BBI+eW are both in fact undecidable.

\section{UNDECIDABILITY OF PROPOSITIONAL FORMULAS FOR THE MEMORY MODELS}

In this section, we give a high-level outline of our undecidability results and their proof, the details of which occupy most of the remainder of the article.

Figure 1 shows the overall development of our proof. As is typical, we show that a property $Q$ is undecidable by showing that a problem already known to be undecidable reduces to the problem of deciding $Q$. In our case, the undecidable problem to be encoded is the termination of a two-counter, non-deterministic Minsky machine $M$ from an arbitrary configuration $C$, shown as the top-centre node in Figure 1.

The proof strategy then goes as follows.

We encode $M$ and $C$ as a formula $\mathcal{F}_{M, C}$ (defined in Section 7 ) such that termination of $M$ from $C$ implies that $\mathcal{F}_{M, C}$ is provable in BBI (Theorem 8.3). In fact, we can obtain this result even for a minimal version of BBI whose Boolean connectives are restricted to $\wedge$ and $\rightarrow$ : in particular, negation $\neg$ and falsum $\perp$ are disallowed. We present this "Minimal BBI" in Section 6.

By construction of the proof systems in Definition 4.1, provability of $\mathcal{F}_{M, C}$ in Minimal BBI implies its provability in BBI and BBI+eW. By our soundness results (Proposition 4.2 and Corollary 4.3) this implies validity of $\mathcal{F}_{M, C}$ in the class of all separation models, and in the class of all separation models with indivisible units, respectively. Specifically, $\mathcal{F}_{M, C}$ must be valid in any particular model chosen from those in Section 2. 


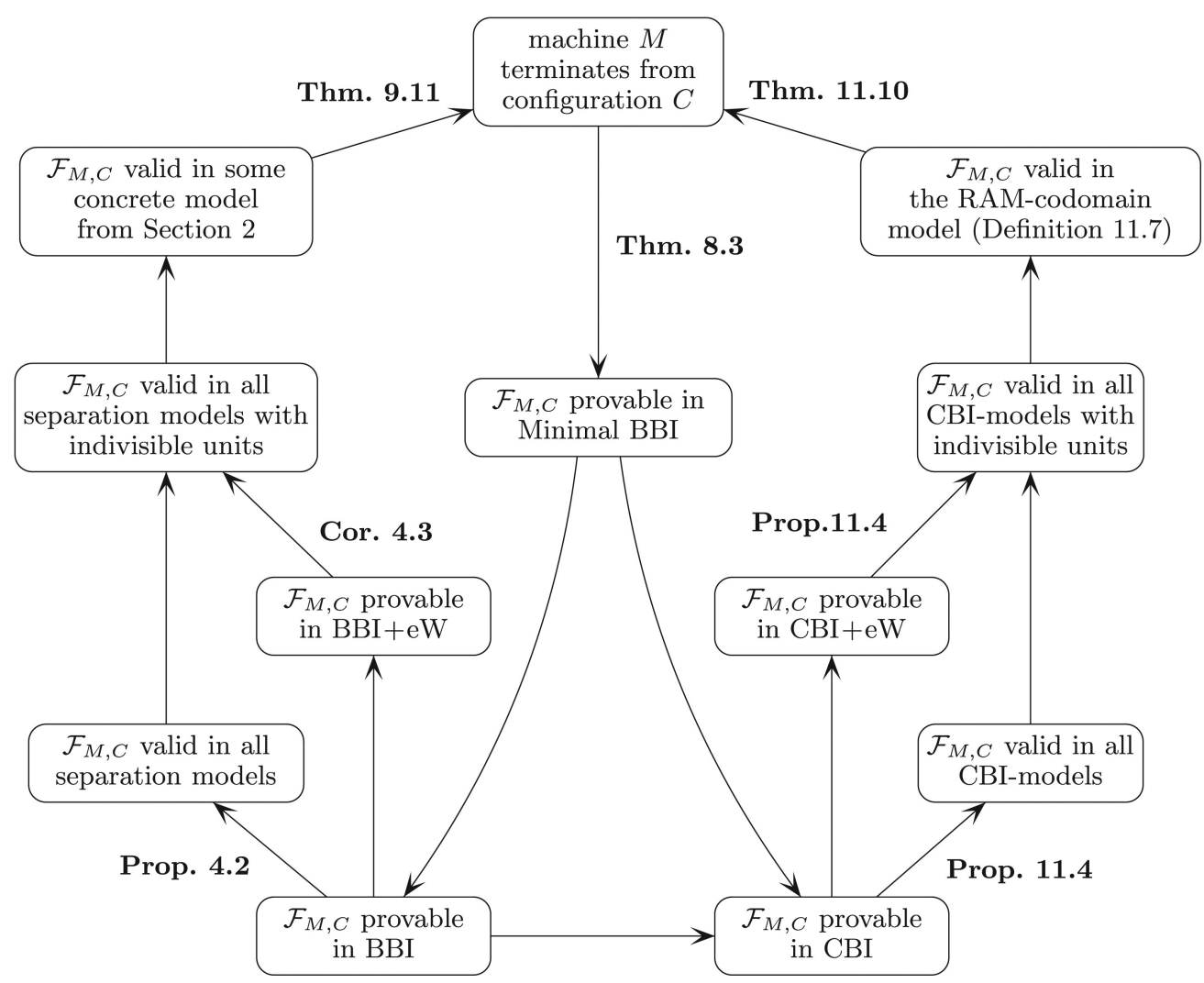

Fig. 1. Diagrammatic proof of undecidability. The arrows are implications, and $\mathcal{F}_{M, C}$ is a sequent built from machine $M$ and configuration $C$. The problems at each node are all undecidable.

Finally, we can complete the circle of implications-and thus the reduction from the halting problem for Minsky machines-by establishing that validity of $\mathcal{F}_{M, C}$ in any particular such model implies that the machine $M$ terminates from configuration $C$. This is established by Theorem 9.11 .

As a consequence, every property of formulas between provability in Minimal BBI and validity in one of the models from Section 2 is undecidable.

The right-hand side of Figure 1 essentially repeats this proof structure, but for Classical BI (CBI) and its models, which obey stronger properties than BBI and its separation models. Classical BI, its models and the corresponding undecidability results are presented in Section 11.

CoROLlary 5.1. The following properties of formulas are undecidable, even when restricted to the language $(\wedge, \rightarrow, \mathrm{I}, *, \rightarrow)$ of Minimal $\mathrm{BBI}$ :

(a) provability in Minimal BBI;

(b) provability in $\mathrm{BBI}$;

(c) provability in $\mathrm{BBI}+\mathrm{eW}$;

(d) validity in the class of all separation models;

(e) validity in the class of all separation models with indivisible units;

$(f)$ validity in the class of all total separation models;

(g) validity in the class of all total separation models with indivisible units; 


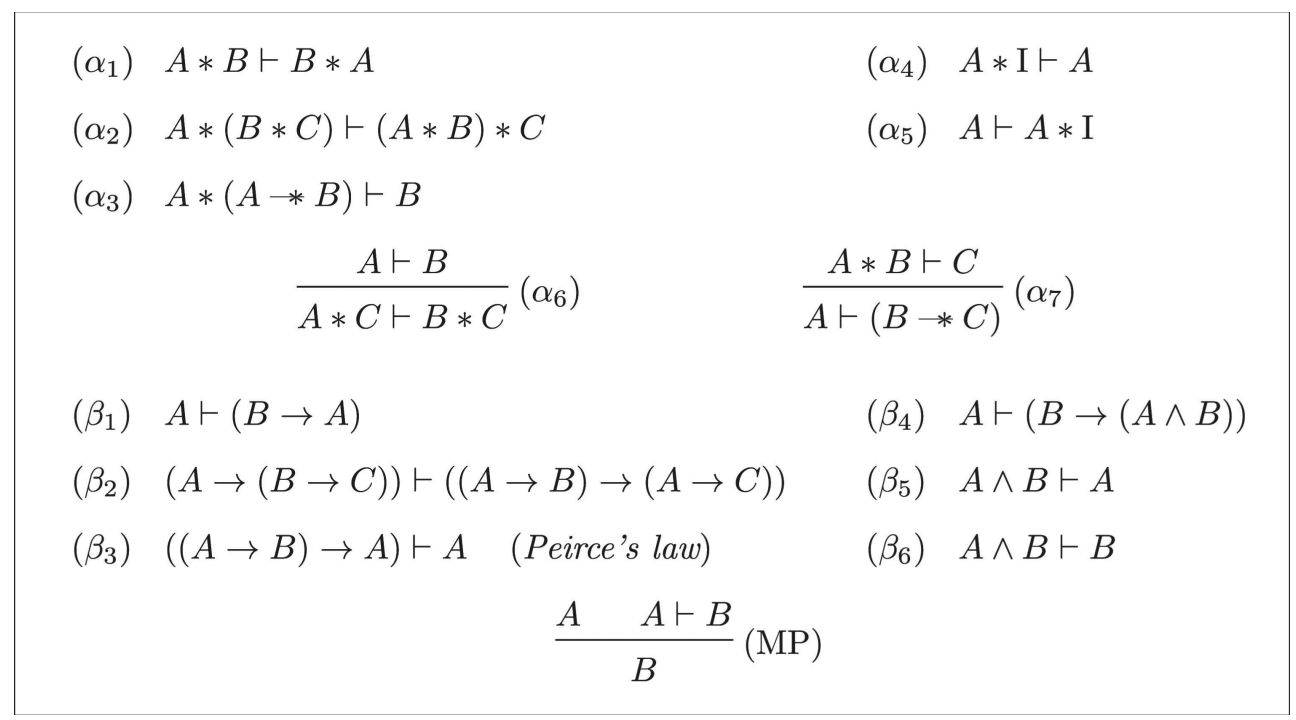

Fig. 2. A minimal set of axioms and rules for Minimal Boolean BI. The axioms and rules labelled $\left(\alpha_{i}\right)$ axiomatize the behaviour of $\mathrm{I}, *$ and $*$, while the axioms and rules labelled $\left(\beta_{i}\right)$ axiomatize $\wedge$ and $\rightarrow$.

(h) validity in one of the concrete models in Section 2, for arbitrarily chosen locations $L$, values $R V$, stacks $S$, and permission algebra $(P, \bullet, \mathbb{1})$ (note L must be infinite).

Proof. The termination problem for Minsky machines, which is undecidable [Minsky 1967], reduces to each of the problems above by the diagram in Figure 1.

Corollary 5.2. Neither Minimal BBI nor BBI nor BBI+eW has the finite model property.

PRoof. A recursive enumeration of proofs and finite countermodels for any of the logics above would yield a decision procedure for provability, which is impossible.

\section{MINIMAL BOOLEAN BI - A VERY SIMPLE UNDECIDABLE PROPOSITIONAL SYSTEM}

One might be tempted to think that the undecidability of BBI, in particular, is mainly an artifact of Boolean negation, which is its most visible point of difference to standard (intuitionistic) BI. In fact, this is not the case. In this section we introduce a minimal positive fragment of BBI, called Minimal BBI, in which the formula connectives are restricted to the minimal set of $\wedge, \rightarrow, \mathrm{I}, *$ and $*$. Minimal BBI is given by the axioms and rules in Figure 2, which extend the axioms and rules for $*, *$ and I in Section 4 (here labelled $\left.\left(\alpha_{i}\right)\right)$ with minimal rules and axioms for $\rightarrow$ and $\wedge$ (labelled $\left(\beta_{i}\right)$ ).

Remark 6.1. As foreshadowed in the previous section, Minimal BBI, even though it looks extremely simple, is still undecidable, notwithstanding the fact that both its components are decidable: the implication-conjunction fragment of Boolean logic is coNP-complete, and intuitionistic multiplicative linear logic is NP-complete [Kanovich 1992].

Oddly enough, removing Peirce's law from Figure 2 results in (a restricted version of) standard intuitionistic BI, which is decidable. This is quite counterintuitive, since by removing Peirce's law we replace the simple Boolean logic component in Figure 2 (given by the $\beta$ axioms and the MP rule) with the implication-conjunction fragment of intuitionistic logic, the complexity of which is higher (being PSPACE-complete [Statman 1979]). 
Note that in Figure 2 we must include the axioms and rules for $\wedge$, since $\wedge$ itself is not definable exclusively in terms of $\rightarrow$. Also, neither $\neg$ nor $\perp$ is expressible by $\wedge$ and $\rightarrow$ alone. As a consequence, it is necessary to establish that various expected principles of classical logic, present in full BBI by construction, still hold in Minimal BBI.

Definition 6.2. As for disjunction, here, and henceforth, we will conceive of $(A \vee B)$ as an abbreviation for $((B \rightarrow A) \rightarrow A)$.

Proposition 6.3. The following inference rules are derivable in Minimal BBI.

$$
\begin{array}{ccc}
\frac{A \vdash B \quad B \vdash C}{A \vdash A}(\mathrm{Id}) & \frac{A \vdash B \quad A \vdash C}{A \vdash C}(\mathrm{Tr}) & \frac{A \vdash B \wedge C}{A \vdash(B \rightarrow C)}(*) \\
\frac{A \vdash(B \rightarrow C)}{A \wedge B \vdash C}(\rightarrow) & \frac{B \vdash C}{A * B \vdash C}(\mathrm{I}) \\
\frac{A \vdash B}{B \rightarrow C \vdash A \rightarrow C}(\mathrm{MT}) & \frac{A \vdash C \quad B \vdash C}{A \vee B \vdash C}(\vee) & \frac{A \vdash C \quad(A \rightarrow B) \vdash C}{C}(\mathrm{EM})
\end{array}
$$

Moreover, the rules $(\rightarrow),(*)$ and $(\mathrm{I})$ are all reversible, that is, their premise and conclusion are interchangeable.

PROOF. We show how to derive each proof rule directly by the standard technique. In the following, recall that the sequent $A \vdash B$ is merely another notation for the formula $A \rightarrow B$.

(Id). By instantiating the axioms $\left(\beta_{1}\right)$ and $\left(\beta_{2}\right)$ we obtain, respectively,

$$
\begin{gathered}
A \vdash((A \rightarrow A) \rightarrow A) \\
(A \rightarrow((A \rightarrow A) \rightarrow A)) \vdash((A \rightarrow(A \rightarrow A)) \rightarrow(A \rightarrow A)) .
\end{gathered}
$$

Thus, by (MP), we obtain $(A \rightarrow(A \rightarrow A)) \vdash(A \rightarrow A)$. Thus, since $A \vdash(A \rightarrow A)$ is an instance of $\left(\beta_{1}\right)$, we obtain $A \vdash A$ by (MP).

(Tr). We have $B \vdash C$ provable by assumption and $(B \rightarrow C) \vdash(A \rightarrow(B \rightarrow C))$ an instance of $\left(\beta_{1}\right)$. Thus, by (MP), we obtain $A \rightarrow(B \rightarrow C)$. Now by instantiating the axiom $\left(\beta_{2}\right)$ we obtain:

$$
(A \rightarrow(B \rightarrow C)) \vdash((A \rightarrow B) \rightarrow(A \rightarrow C)),
$$

whence by $(\mathrm{MP})$ we obtain $(A \rightarrow B) \vdash(A \rightarrow C)$. Then, since $A \vdash B$ is provable by assumption we obtain $A \vdash C$ by (MP) again as required.

$(\wedge)$. We have $A \vdash B$ by assumption and $B \vdash(C \rightarrow B \wedge C)$ an instance of $\left(\beta_{4}\right)$. Thus, by (Tr), we obtain $A \vdash(C \rightarrow B \wedge C)$. Now, by instantiating $\left(\beta_{2}\right)$ we have:

$$
(A \rightarrow(C \rightarrow B \wedge C)) \vdash((A \rightarrow C) \rightarrow(A \rightarrow B \wedge C)) .
$$

Hence, by (MP), we obtain $(A \rightarrow C) \vdash(A \rightarrow B \wedge C)$. Since we have $A \vdash C$, by assumption, we obtain $A \vdash B \wedge C$ by (MP) as required.

$(\rightarrow)$. For the top-to-bottom direction, note that we have $A \wedge B \vdash A$ an instance of $\left(\beta_{5}\right)$ and $A \vdash(B \rightarrow C)$ provable by assumption. Thus, by (Tr), we obtain $A \wedge B \vdash(B \rightarrow C)$. Now, by instantiating $\left(\beta_{2}\right)$, we have:

$$
(A \wedge B \rightarrow(B \rightarrow C)) \vdash((A \wedge B \rightarrow B) \rightarrow(A \wedge B \rightarrow C)) .
$$


Hence, by (MP), we obtain $(A \wedge B \rightarrow B) \vdash(A \wedge B \rightarrow C)$. Since $A \wedge B \vdash B$ is an instance of $\left(\beta_{6}\right)$, we can apply (MP) again to obtain $A \wedge B \vdash C$ as required.

For the bottom-to-top direction, we have $A \wedge B \vdash C$ by assumption. Thus, using $\left(\beta_{1}\right)$ and $(\mathrm{Tr})$, we obtain $B \vdash(A \wedge B \rightarrow C)$. By instantiating $\left(\beta_{2}\right)$, we have

$$
B \rightarrow((A \wedge B) \rightarrow C) \vdash((B \rightarrow A \wedge B) \rightarrow(B \rightarrow C)) .
$$

Hence, by (MP), we obtain $(B \rightarrow A \wedge B) \vdash(B \rightarrow C)$. Applying $\left(\beta_{1}\right)$ and (Tr) again, this gives us $A \vdash((B \rightarrow A \wedge B) \rightarrow(B \rightarrow C))$. Now we have as a further instantiation of $\left(\beta_{2}\right)$

$$
A \rightarrow((B \rightarrow A \wedge B) \rightarrow(B \rightarrow C)) \vdash((A \rightarrow(B \rightarrow A \wedge B)) \rightarrow(A \rightarrow(B \rightarrow C))) .
$$

Hence, by (MP), we obtain

$$
(A \rightarrow(B \rightarrow A \wedge B)) \vdash(A \rightarrow(B \rightarrow C)) .
$$

As $A \vdash B \rightarrow A \wedge B$ is an instance of $\left(\beta_{4}\right)$, we obtain $A \vdash B \rightarrow C$ by (MP) as required.

(*). The bottom-to-top direction is immediate by $\left(\alpha_{7}\right)$. For the top-to-bottom direction, since $A \vdash(B * C)$ is provable by assumption, we have $A * B \vdash(B * C) * B$ by the rule $\left(\alpha_{6}\right)$. Since $(B * C) * B \vdash B *(B * C)$ is an instance of $\left(\alpha_{1}\right)$, we then obtain $A * B \vdash B *(B * C)$ by (Tr). Then, since $B *(B * C) \vdash C$ is an instance of $\left(\alpha_{3}\right)$, we can apply ( $\left.\mathrm{Tr}\right)$ again to obtain $A * B \vdash C$ as required.

(I). For the top-to-bottom direction, we have $B * I \vdash B$ an instance of $\left(\alpha_{4}\right)$ and $I * B \vdash B * I$ an instance of $\left(\alpha_{1}\right)$ whence $I * B \vdash B$ is provable by (Tr). Since $B \vdash C$ is provable by assumption, we obtain $I * B \vdash C$ by (Tr), whence $I \vdash(B * C)$ is provable using the rule $(*)$. The reverse direction is similar.

(MT). (This rule is named for being "modus tollens-like".) First, $(B \rightarrow C) \vdash(B \rightarrow C)$ is derivable using (Id). Thus, by the rule $(\rightarrow)$, together with $(\mathrm{Tr})$ and commutativity of $\wedge$ (easily derivable from the rule $(\wedge)$ ), we obtain $B \vdash((B \rightarrow C) \rightarrow C)$. Since we have $A \vdash B$ by assumption, we obtain $A \vdash((B \rightarrow C) \rightarrow C)$ by (Tr). Thus, by applying the deduction theorem again, together with commutativity of $\wedge$ and (Tr), we have $(B \rightarrow C) \vdash(A \rightarrow C)$ as required.

( $\vee$ ). Since we have $B \vdash C$ by assumption, we can obtain by applying the rule (MT) twice

$$
((B \rightarrow A) \rightarrow A) \vdash((C \rightarrow A) \rightarrow A) .
$$

By applying the rule $(\rightarrow)$, we then obtain $(B \rightarrow A) \rightarrow A) \wedge(C \rightarrow A) \vdash A$. Since $A \vdash C$ is provable by assumption, we obtain $(B \rightarrow A) \rightarrow A) \wedge(C \rightarrow A) \vdash C$ by applying (Tr). By applying $(\rightarrow)$ again, we have

$$
((B \rightarrow A) \rightarrow A) \vdash((C \rightarrow A) \rightarrow C) .
$$

Now we have $((C \rightarrow A) \rightarrow C) \vdash C$ an instance of Peirce's law $\left(\beta_{3}\right)$. Thus, we obtain $((B \rightarrow A) \rightarrow A) \vdash C$ by (Tr), which is abbreviated by $A \vee B \vdash C$.

(EM). (This rule is named for being "excluded middle-like".) We have by assumption $A \vdash C$ and $(A \rightarrow B) \vdash C$, so we obtain $A \vee(A \rightarrow B) \vdash C$ by applying the rule $(\vee)$. By definition, $A \vee(A \rightarrow B)$ is equal to $((A \rightarrow B) \rightarrow A) \vdash A$, which is an instance of Peirce's law $\left(\alpha_{3}\right)$. Thus, we obtain $C$ by (MP). 
We remark that the rules $(\wedge)$ and $(\vee)$ given by Proposition 6.3 immediately imply the associativity and commutativity of $\wedge$ and $\vee$, whereas associativity and commutativity of $*$ is directly expressed by axioms $\left(\alpha_{1}\right)$ and $\left(\alpha_{2}\right)$ of Minimal BBI. From now on, when we work in Minimal BBI, we implicitly treat $\wedge, \vee$, and $*$ as being commutative and associative.

As mentioned in Section 4, the restricted $*$-contraction given by $I \wedge A \vdash A * A$ holds in BBI, and in fact it holds even in Minimal BBI, which is of utmost importance for our undecidability results. This principle is obviously semantically valid, since for any $e \in E$, we have $e=e \circ e$ and thus if $e \models_{\rho} A$ then $e \models_{\rho} A * A$.

However, it is far from obvious that it is provable in BBI, and still less so in Minimal BBI. The broad idea of the BBI proof is as follows. First, from $\mathrm{I} \wedge A$ we can deduce $((\mathrm{I} \wedge A) * \mathrm{I})$ and thus $(\mathrm{I} \wedge A) *(\mathrm{I} \wedge(A \vee \neg A))$ using the usual law of excluded middle in classical logic. Since $\vee$ distributes over both $\wedge$ and $*$, the latter sequent yields $((\mathrm{I} \wedge A) *(\mathrm{I} \wedge A)) \vee((\mathrm{I} \wedge A) *(\mathrm{I} \wedge \neg A))$ but $(\mathrm{I} \wedge A) *(\mathrm{I} \wedge \neg A)$ implies $A \wedge \neg A$, which is inconsistent.

In Minimal BBI, the situation is complicated further by the absence of $\neg$ in the language, forcing us to use Peirce's law, in the shape of the rule (EM). The following lemma spells out the full details.

LEMMA 6.4. The restricted *-contraction, given by a sequent of the form

$$
\mathrm{I} \wedge A \vdash A * A
$$

is provable in Minimal BBI.

Proof. We make free use of the derived rules of Minimal BBI given by Proposition 6.3. According to the reversible version of the rule (I), the sequent (11) follows from

$$
\mathrm{I} \vdash(\mathrm{I} \wedge A) *(A * A),
$$

which is notation for the formula

$$
\mathrm{I} \rightarrow((\mathrm{I} \wedge A) *(A * A)) .
$$

Using the "excluded middle" rule (EM) in Proposition 6.3, we can derive the formula (12) from the following two sequents:

$$
A \vdash \mathrm{I} \rightarrow((\mathrm{I} \wedge A) *(A * A))
$$

and

$$
A \rightarrow(A * A) \vdash \mathrm{I} \rightarrow((\mathrm{I} \wedge A) \rightarrow(A * A)) .
$$

We proceed by demonstrating how to derive each of (13) and (14).

-We derive the sequent (13) as follows. First, using the fact that $\mathrm{I} \wedge A \vdash A\left(\operatorname{axiom}\left(\beta_{6}\right)\right)$ and the rule $\left(\alpha_{6}\right)$ for $*$ we have:

$$
(\mathrm{I} \wedge A) *(\mathrm{I} \wedge A) \vdash A *(\mathrm{I} \wedge A) .
$$

By a similar sequence of reasoning, but additionally using commutativity of $*\left(\alpha_{1}\right)$, we have $A *(\mathrm{I} \wedge A) \vdash A * A$, whereby we have by $(\mathrm{Tr})$

$$
(\mathrm{I} \wedge A) *(\mathrm{I} \wedge A) \vdash A * A \text {. }
$$

Using the derived rule $(*)$, we obtain:

$$
\mathrm{I} \wedge A \vdash((\mathrm{I} \wedge A) \rightarrow(A * A))
$$

and, by means of the derived rule $(\rightarrow)$, we conclude with the desired sequent (13). 
-We derive the sequent (14) in the following way. First, using $\wedge$-weakening $\left(\beta_{5}\right),\left(\beta_{6}\right)$ and the axiom $\left(\alpha_{4}\right)$ for I, we can derive each of the following:

$$
(\mathrm{I} \wedge(A \rightarrow(A * A))) *(\mathrm{I} \wedge A) \vdash \mathrm{I} *(\mathrm{I} \wedge A)
$$

and

$$
\mathrm{I} *(\mathrm{I} \wedge A) \vdash A,
$$

whence by transitivity $(\mathrm{Tr})$ we obtain:

$$
(\mathrm{I} \wedge(A \rightarrow(A * A))) *(\mathrm{I} \wedge A) \vdash A .
$$

Next, using the same principles, we can derive

$$
(\mathrm{I} \wedge(A \rightarrow(A * A))) *(\mathrm{I} \wedge A) \vdash(\mathrm{I} \wedge(A \rightarrow(A * A))) * \mathrm{I},
$$

and

$$
(\mathrm{I} \wedge(A \rightarrow(A * A))) * \mathrm{I} \vdash(A \rightarrow(A * A)),
$$

resulting by transitivity $(\mathrm{Tr})$ in

$$
(\mathrm{I} \wedge(A \rightarrow(A * A))) *(\mathrm{I} \wedge A) \vdash(A \rightarrow(A * A)) .
$$

Using the axiom $\left(\beta_{2}\right)$ from Figure 2 and the derived rules $(\wedge),(\rightarrow)$ and $(\mathrm{Tr})$, it is straightforward to derive from (15) and (16):

$$
(\mathrm{I} \wedge(A \rightarrow(A * A))) *(\mathrm{I} \wedge A) \vdash A * A .
$$

Hence, by applying the derived rules $(*)$, we obtain

$$
\mathrm{I} \wedge(A \rightarrow(A * A)) \vdash((\mathrm{I} \wedge A) *(A * A))
$$

and, by means of the derived rules $(\rightarrow)$, we conclude with the desired sequent (14).

The proof principles of Proposition 6.3 and Lemma 6.4 are the main ones that are needed for correctness of our encoding of Minsky machines in the next section.

\section{ENCODING MINSKY MACHINE COMPUTATIONS IN MINIMAL BBI}

In this section, we give our encoding of terminating computations of (nondeterministic) two-counter Minsky machines as provable sequents of Minimal BBI. That is to say, given a Minsky machine $M$ and initial configuration $C$ our aim is to give a sequent $\mathcal{F}_{M, C}$ such that $M$ terminates from $C$ just in case $\mathcal{F}_{M, C}$ is provable in Minimal BBI (as per the strategy in Section 5). First, we explain how $\mathcal{F}_{M, C}$ is constructed from $M$ and $C$, and then in Section 7.1 we develop some intuition for our choice of encoding.

Definition 7.1. A nondeterministic, two-counter Minsky machine $M$ [Minsky 1967] with non-negative counters $c_{1}, c_{2}$ is given by a finite set of labelled instructions of the form:

$$
\begin{array}{ll}
\text { “increment } c_{k} \text { by } 1 \text { " } & L_{i}: c_{k}++ \text {; goto } L_{j} ; \\
\text { "decrement } c_{k} \text { by } 1 \text { " } & L_{i}: c_{k}--; \text { goto } L_{j} ; \\
\text { “zero-test on } c_{k} \text { " } & L_{i} \text { : if } c_{k}=0 \text { goto } L_{j} ; \\
\text { "goto" } & L_{i} \text { : goto } L_{j} ;
\end{array}
$$

where $k \in\{1,2\}, i \geq 1$ and $j \geq 0$. 


$$
\begin{aligned}
& \frac{\left(L_{i}: c_{1}++; \text { goto } L_{j} ;\right) \in M}{\left\langle L_{i}, n_{1}, n_{2}\right\rangle \sim_{M}\left\langle L_{j}, n_{1}+1, n_{2}\right\rangle} \\
& \frac{\left(L_{i}: c_{2}++; \text { goto } L_{j} ;\right) \in M}{\left\langle L_{i}, n_{1}, n_{2}\right\rangle \leadsto M\left\langle L_{j}, n_{1}, n_{2}+1\right\rangle} \\
& \frac{\left(L_{i}: c_{1}--; \text { goto } L_{j} ;\right) \in M}{\left\langle L_{i}, n_{1}+1, n_{2}\right\rangle \sim M\left\langle L_{j}, n_{1}, n_{2}\right\rangle} \\
& \frac{\left(L_{i}: c_{2}--; \text { goto } L_{j} ;\right) \in M}{\left\langle L_{i}, n_{1}, n_{2}+1\right\rangle \sim M\left\langle L_{j}, n_{1}, n_{2}\right\rangle}
\end{aligned}
$$

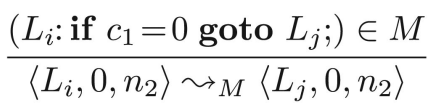

$$
\begin{aligned}
& \frac{\left(L_{i}: \text { if } c_{2}=0 \text { goto } L_{j} ;\right) \in M}{\left\langle L_{i}, n_{1}, 0\right\rangle \sim_{M}\left\langle L_{j}, n_{1}, 0\right\rangle} \\
& \frac{\left(L_{i} \text { : goto } L_{j} ;\right) \in M}{\left\langle L_{i}, n_{1}, n_{2}\right\rangle \sim_{M}\left\langle L_{j}, n_{1}, n_{2}\right\rangle}
\end{aligned}
$$

Fig. 3. One-step semantics of two-counter Minsky machines.

Nondeterminism of $M$ means that each "state label" $L_{i}$ may have multiple associated instructions. The labels $L_{0}$ and $L_{1}$ are reserved for the final and initial states of $M$, respectively. In particular, $L_{0}$ may not label any instructions.

To cope more easily with zero-test instructions, we also add the special labels $L_{-1}$ and $L_{-2}$ which come equipped with the following four instructions (and which may not label any other instructions).

$$
\begin{array}{ll}
L_{-1}: c_{2}--; \text { goto } L_{-1} ; & L_{-1}: \text { goto } L_{0} ; \\
L_{-2}: c_{1}--; \text { goto } L_{-2} ; & L_{-2}: \text { goto } L_{0} ;
\end{array}
$$

A configuration of $M$ is given by $\left\langle L, n_{1}, n_{2}\right\rangle$, where the label $L$ is the current state of $M$, and $n_{1}$ and $n_{2}$ are the current values of counters $c_{1}$ and $c_{2}$, respectively.

We write $\sim_{M}$ for the one-step relation between configurations of $M$; the one-step semantics of Minsky machines is given by Figure 3. We let $\sim_{M}^{*}$ be the reflexive-transitive closure of $\sim_{M}$, so that $\left\langle L, n_{1}, n_{2}\right\rangle \sim_{M}^{*}\left\langle L^{\prime}, n_{1}^{\prime}, n_{2}^{\prime}\right\rangle$ if $M$ can go from $\left\langle L, n_{1}, n_{2}\right\rangle$ to $\left\langle L^{\prime}, n_{1}^{\prime}, n_{2}^{\prime}\right\rangle$ in a finite number of steps. If $\left\langle L, n_{1}, n_{2}\right\rangle \sim_{M}^{*}\left\langle L_{0}, 0,0\right\rangle$, we say that $M$ terminates from $\left\langle L, n_{1}, n_{2}\right\rangle$, written $\left\langle L, n_{1}, n_{2}\right\rangle \Downarrow_{M}$.

The specific role of the special labels $L_{-1}$ and $L_{-2}$ is explained by the following lemma.

LEMMA 7.2. $\left\langle L_{-k}, n_{1}, n_{2}\right\rangle \Downarrow_{M}$ if and only if $n_{k}=0$, where $k \in\{1,2\}$.

Proof. Formally, the proof proceeds by induction on $n_{2}$ if $k=1$, and vice versa; we just provide a sketch here since the details are straightforward. For the "if" direction, we simply apply the relevant instructions from the group (18). For the "only if" direction, we simply notice that, by construction, no other instructions except those in the group (18) are applicable to any configuration of the form $\left\langle L_{-k}, n_{1}, n_{2}\right\rangle$.

Definition 7.3. In our encoding, we use the following abbreviation. We fix an atomic proposition $b$, and henceforth define a "relative negation" by: $-A=_{\text {def }}(A * b)$.

Our relative negation, defined as multiplicative implication into a constant, can be seen as a natural multiplicative analogue of standard intuitionistic negation, which is defined by $\neg A=A \rightarrow \perp$. We derive some useful properties of relative negation in Lemma 8.1. 
Definition 7.4 (Machine Encoding). For any instruction $\gamma$ from Definition 7.1, we define the formula $\kappa(\gamma)$ as follows:

$$
\begin{aligned}
\kappa\left(L_{i}: c_{k}++; \text { goto } L_{j} ;\right) & =_{\text {def }}\left(-\left(l_{j} * p_{k}\right) *-l_{i}\right) \\
\kappa\left(L_{i}: c_{k}--; \text { goto } L_{j} ;\right) & =_{\text {def }}\left(-l_{j} *-\left(l_{i} * p_{k}\right)\right) \\
\kappa\left(L_{i}: \text { if } c_{k}=0 \text { goto } L_{j} ;\right) & =_{\text {def }}\left(-\left(l_{j} \vee l_{-k}\right) *-l_{i}\right) \\
\kappa\left(L_{i}: \text { goto } L_{j} ;\right) & =_{\text {def }}\left(-l_{j} *-l_{i}\right),
\end{aligned}
$$

where $p_{1}, p_{2}, l_{-2}, l_{-1}, l_{0}, l_{1}, l_{2}, \ldots$ are distinct atomic propositions ( $p_{1}$ and $p_{2}$ are used to represent the counters $c_{1}$ and $c_{2}$, respectively).

Recall that, by Definition 6.2, the formula $\left(-\left(l_{j} \vee l_{-k}\right) *-l_{i}\right)$ abbreviates the formula $\left(-\left(\left(l_{-k} \rightarrow l_{j}\right) \rightarrow l_{j}\right) \rightarrow-l_{i}\right)$.

Then, for a Minsky machine $M$ given by instructions $\left\{\gamma_{1}, \gamma_{2}, \ldots, \gamma_{t}\right\}$, we define its encoding formula $\kappa(M)$ by

$$
\kappa(M)==_{\text {def }} \mathrm{I} \wedge \bigwedge_{i=1}^{t} \kappa\left(\gamma_{i}\right) .
$$

Definition 7.5 (Encoding of Terminating Configurations). We will encode nonnegative integers $n$, the contents of a Minsky machine counter, as a formula of the form

$$
p^{n}=_{\text {def }} \underbrace{p * p * \cdots * p}_{n \text { times }}
$$

with $p^{0}=_{\text {def }} \mathrm{I}$.

Accordingly, we encode a machine configuration of the form

$$
C=\left\langle L_{i}, n_{1}, n_{2}\right\rangle
$$

as a $*$-conjunction of the propositional variable $l_{i}$ with $n_{1}$ copies of $p_{1}$ and $n_{2}$ copies of $p_{2}$ :

$$
l_{i} * p_{1}^{n_{1}} * p_{2}^{n_{2}}
$$

The fact that $\left\langle L_{0}, 0,0\right\rangle$ is a terminating configuration is encoded by the following formula:

$$
\mathrm{I} \wedge-l_{0} \text {. }
$$

Putting everything together, termination of $M$ from $\left\langle L_{i}, n_{1}, n_{2}\right\rangle$ will be encoded by the following sequent $\mathcal{F}_{M, C}$ :

$$
\mathcal{F}_{M, C}=_{\text {def }} \kappa(M) * l_{i} * p_{1}^{n_{1}} * p_{2}^{n_{2}} *\left(\mathrm{I} \wedge-l_{0}\right) \vdash b,
$$

where $\kappa(M)$ is the machine encoding given by Definition 7.4 and $b$ is the fixed proposition letter used in our "relative negation" (Definition 7.3).

\subsection{Intuition for Our Encoding}

In accordance with our proof strategy in Section 5, our encoding is intended to achieve two complementary goals.

(1) On one hand, we have to show provability (in Minimal BBI) of $\mathcal{F}_{M, C}$ whenever $M$ terminates from $C$. We construct the proof of $\mathcal{F}_{M, C}$ by induction on the length $m$ of the terminating computation, using as subproofs the proofs given by induction for terminating computations of shorter length. We give the full details of this construction in Section 8. 
(2) On the other hand, for a fixed memory model from Section 2, we have to find a valuation $\rho$ such that $M$ terminates from $C$ of the form $\left\langle L_{i}, n_{1}, n_{2}\right\rangle$ whenever $\mathcal{F}_{M, C}$ is valid under this valuation $\rho$, that is, whenever

$$
\llbracket \kappa(M) * l_{i} * p_{1}^{n_{1}} * p_{2}^{n_{2}} *\left(\mathrm{I} \wedge-l_{0}\right) \rrbracket_{\rho} \subseteq \rho(b) .
$$

To obtain the required termination of $M$ from $C$ from this inclusion, it stands to reason to define $\rho(b)$ so as to represent all terminating configurations, and then to show that

$$
e \in \llbracket \kappa(M) \rrbracket_{\rho} \quad \text { and } \quad e \in \llbracket \mathrm{I} \wedge-l_{0} \rrbracket_{\rho},
$$

where $e$ is a unit of the memory model, resulting in the inclusion:

$$
\llbracket l_{i} * p_{1}^{n_{1}} * p_{2}^{n_{2}} \rrbracket_{\rho} \subseteq \rho(b)
$$

Provided that each element of $\llbracket l_{i} * p_{1}^{n_{1}} * p_{2}^{n_{2}} \rrbracket_{\rho}$ uniquely determines the configuration $\left\langle L_{i}, n_{1}, n_{2}\right\rangle$ (cf. Lemma 9.2), we can then deduce that $\left\langle L_{i}, n_{1}, n_{2}\right\rangle \Downarrow_{M}$.

However, an additional twist to the problem is that we need a rather complicated valuation $\rho$ in the memory models of Section 2 , because of the way the partial composition is defined in these models. We look at this point in more detail in Sections 9.2 and 10 .

We note that a direct adaptation of the encoding of Minsky machines developed for full linear logic in Kanovich [1995] does not work properly for separation logic due to the differences between linear logic and separation logic (discussed further in Section 12).

Roughly speaking, in the encoding of Kanovich [1995], each step in the derivation of the "encoding sequent" corresponds to a single forward step from $C$ to $C^{\prime}$ within the computation of machine $M$ (which is not necessarily terminating).

In contrast, in our encoding, each step in the derivation corresponds to a "backward move" from the class of all terminating computations starting from $C^{\prime}$ to the class of longer terminating computations starting from $C$.

We illustrate this point in more detail.

Given a memory model, suppose that a valuation $\rho$ is chosen such that each element of $\llbracket l_{i} * p_{1}^{n_{1}} * p_{2}^{n_{2}} \rrbracket_{\rho}$ uniquely determines the configuration $\left\langle L_{i}, n_{1}, n_{2}\right\rangle$ (cf. Lemma 9.2).

As discussed previously, to guarantee the faithfulness of our encoding, we need to check that for such a valuation $\rho$, the encoding guarantees that $e \in \llbracket \kappa(M) \rrbracket_{\rho}$, that is, for each of the machine instruction $\gamma$ we can provide $e \in \llbracket \kappa(\gamma) \rrbracket_{\rho}$.

For any instruction $\gamma$, the formula $\kappa(\gamma)$ is of the form $(-A *-B)$ where $A$ is of the form $l_{j},\left(l_{j} \vee l_{-k}\right)$ or $\left(l_{j} * p_{k}\right)$, and $B$ is of the form $l_{i}$ or $\left(l_{i} * p_{k}\right)$. Thus, recalling that $-A=_{\text {def }}(A * b)$, and $-B=_{\text {def }}(B * b)$, the statement $e \in \llbracket \kappa(\gamma) \rrbracket_{\rho}$ is equivalent to the inclusion

$$
\llbracket A * b \rrbracket_{\rho} \subseteq \llbracket B * b \rrbracket_{\rho} .
$$

Since, by Lemma 3.8, the statement $d \in \llbracket A \rightarrow b \rrbracket_{\rho}$ means that $\llbracket A \rrbracket_{\rho} \cdot\{d\} \subseteq \rho(b)$, this inclusion can be rewritten as the rule that for all $d$,

$$
\frac{\llbracket A \rrbracket_{\rho} \cdot\{d\} \subseteq \rho(b)}{\llbracket B \rrbracket_{\rho} \cdot\{d\} \subseteq \rho(b)} .
$$

For instance, in the case of the increment instruction

$$
L_{i}: c_{1}++ \text {; goto } L_{j}
$$

we must show that the following rule is correct for any $d$ :

$$
\frac{\llbracket l_{j} * p_{1} \rrbracket_{\rho} \cdot\{d\} \subseteq \rho(b)}{\llbracket l_{i} \rrbracket_{\rho} \cdot\{d\} \subseteq \rho(b)} .
$$


Our choice of valuation (cf. Lemma 9.2) ensures that if $\llbracket l_{j} * p_{1} \rrbracket_{\rho} \cdot\{d\} \subseteq \rho(b)$, then $d$ must belong to a set of the form $\llbracket p_{1}^{n_{1}} * p_{2}^{n_{2}} \rrbracket_{\rho}$, so that this rule becomes

$$
\frac{\llbracket l_{j} * p_{1}^{n_{1}+1} * p_{2}^{n_{2}} \rrbracket_{\rho} \subseteq \rho(b)}{\llbracket l_{i} * p_{1}^{n_{1}} * p_{2}^{n_{2}} \rrbracket_{\rho} \subseteq \rho(b)}
$$

or, paraphrasing,

$$
\frac{\text { " } M \text { terminates from the configuration }\left\langle L_{j}, n_{1}+1, n_{2}\right\rangle "}{\text { " } M \text { terminates from the configuration }\left\langle L_{i}, n_{1}, n_{2}\right\rangle "},
$$

which is a correct statement according to the increment instruction (20) at hand. Other instructions are treated similarly.

\section{FROM COMPUTATIONS TO MINIMAL BBI PROOFS}

Here, we show in detail that the sequent $\mathcal{F}_{M, C}$, defined in the previous section, is provable in Minimal BBI whenever $M$ terminates on $C$. The converse direction will be established in Section 9.

First, the following lemma derives useful properties of - $A$ which illustrate its behaviour as a multiplicative analogue of intuitionistic negation, and will be essential to the proof of our main result.

LEMMA 8.1. The following sequents and proof rules are derivable in Minimal BBI.

(a) $A \vdash--A$

(b) $---A \vdash-A$

(c) $A *(-B *-A) \vdash--B$

(d) $\frac{A * B \vdash C}{A *-B \vdash--C}$

(e) $\frac{A * B \vdash D \quad A * C \vdash D}{A *--(B \vee C) \vdash--D}$

PRoof. We derive each sequent or proof rule directly in Minimal BBI, making use of the derived rules given by Proposition 6.3.

(a) According to the Minimal BBI axiom $\left(\alpha_{3}\right)$, we have $A *(A * b) \vdash b$ provable. Thus, by applying the derived rule $(*)$, we obtain $A \vdash((A * b) * b)$, that is, $A \vdash--A$, as required.

(b) According to the Minimal BBI axiom $\left(\alpha_{3}\right)$, we have $--A *(--A * b) \vdash b$ provable. Also, $A \vdash--A$ is provable by part (a). Thus, by the derived rule (Tr), we obtain $A *(--A * b) \vdash b$. By applying (*) again, we obtain $(--A * b) \vdash A * b$, i.e. $--A \vdash-A$, as required.

(c) We have $-B *(-B *-A) \vdash-A$ an instance of the Minimal BBI axiom $\left(\alpha_{3}\right)$, which can then be rewritten as $-B *(-B *-A) \vdash A * b$. By applying (*), we obtain $A *(-B *-A) \vdash-B \rightarrow b$, that is, $A *(-B *-A) \vdash--B$, as required.

(d) We have $A * B \vdash C$ by assumption and $C \vdash--C$ by part (a), whence by (Tr) we obtain $A * B \vdash--C$. By applying ( $*$ ), we obtain $A *-C \vdash-B$. Since $-B \vdash---B$ is derivable by part (a), we obtain $A *-C \vdash---B$ by (Tr). By applying ( $*$ ) again, this can be transformed to the required $A *--B \vdash--C$.

(e) By applying ( $*$ ) to the premises $A * B \vdash D$ and $A * C \vdash D$, we have $B \vdash(A * D)$ and $C \vdash(A * D)$. Thus, by applying the derived rule $(\vee)$, we obtain $B \vee C \vdash(A \rightarrow D)$. Applying ( $*)$, we get $A *(B \vee C) \vdash D$. Finally, we apply the derived rule given by part (d) to obtain $A *--(B \vee C) \vdash--D$ as required. 
In the process of computation, each instruction of a Minsky machine can be reused an unlimited number of times. In order for us to simulate Minsky computations within Minimal BBI proofs, it is essential that the formulas $\kappa(\gamma)$ representing reusable instructions can be duplicated as needed, which follows from the restricted $*$-contraction given by Lemma 6.4 .

LemMa 8.2. For each instruction $\gamma$ of a machine $M$, the sequent

$$
\kappa(M) \vdash \kappa(M) * \kappa(\gamma)
$$

is derivable in Minimal BBI.

Proof. Writing $M=\left\{\gamma_{1}, \gamma_{2}, \ldots, \gamma_{t}\right\}$, we have $\kappa(M)=\left(\mathrm{I} \wedge \wedge_{i=1}^{t} \kappa\left(\gamma_{i}\right)\right)$ and $\gamma=\gamma_{j}$ for some $1 \leq j \leq t$. First, using the derived rules $(\mathrm{Id})$ and $(\wedge)$ and the axiom $\left(\beta_{5}\right)$ for $\wedge$, we can derive

$$
\kappa(M) \vdash \mathrm{I} \wedge \kappa(M) .
$$

Next, we have as an instance of restricted $*$-contraction (Lemma 6.4)

$$
\mathrm{I} \wedge \kappa(M) \vdash \kappa(M) * \kappa(M) .
$$

We have $\kappa(M) \vdash \kappa\left(\gamma_{j}\right)$ derivable in Minimal BBI by using weakening for $\wedge$ (given by the axioms $\left(\beta_{5}\right)$ and $\left.\left(\beta_{6}\right)\right)$ and the derived transitivity rule (Tr) of Proposition 6.3. Thus, by applying the rule $\left(\alpha_{6}\right)$, we obtain

$$
\kappa(M) * \kappa(M) \vdash \kappa(M) * \kappa\left(\gamma_{j}\right)
$$

Thus, using the transitivity rule $(\mathrm{Tr})$, we obtain $\kappa(M) \vdash \kappa(M) * \kappa\left(\gamma_{j}\right)$ as required.

THeORem 8.3. Let $M$ be a Minsky machine, and for a configuration $C$ of the form $\left\langle L_{i}, n_{1}, n_{2}\right\rangle$, suppose that $M$ terminates from $C$. Then, the following sequent $\mathcal{F}_{M, C}$ is derivable in Minimal BBI:

$$
\kappa(M) * l_{i} * p_{1}^{n_{1}} * p_{2}^{n_{2}} *\left(\mathrm{I} \wedge-l_{0}\right) \vdash b .
$$

Proof. We make free use of the derived rules of Minimal BBI given by Proposition 6.3. First, we show that it suffices to derive the sequent:

$$
\kappa(M) * l_{i} * p_{1}^{n_{1}} * p_{2}^{n_{2}} \vdash--l_{0} .
$$

To see this, suppose we have $A \vdash--l_{0}$, whence by applying the derived rule ( $*$, we obtain $-l_{0} \vdash(A * b)$. Since $\mathrm{I} \wedge-l_{0} \vdash-l_{0}$ is an instance of the axiom $\left(\beta_{6}\right)$, we obtain $\mathrm{I} \wedge-l_{0} \vdash(A * b)$ by applying the derived rule (Tr). By applying (*) again, we obtain $A *\left(\mathrm{I} \wedge-l_{0}\right) \vdash b$ as required.

We show that (22) is derivable in Minimal BBI by induction on the length $m$ of the computation of $\left\langle L_{i}, n_{1}, n_{2}\right\rangle \sim{ }_{M}^{*}\left\langle L_{0}, 0,0\right\rangle$. In the base case $m=0$, we have $n_{1}=n_{2}=0$, and must derive:

$$
\kappa(M) * l_{0} * \mathrm{I} * \mathrm{I} \vdash--l_{0} .
$$

This is easily derivable using the equivalence $I * A \equiv A$, weakening for $\wedge$ and part (a) of Lemma 8.1.

Next, we assume that the result holds for all computations of length $m-1$, and show that it holds for any computation of length $m$. We then proceed by case distinction on the instruction $\gamma$ which yields the first step of the computation. We show the cases for a goto instruction, and for increment, decrement and zero-test instructions with the counter variable $k=1$; the cases for $k=2$ are similar.

Case $\gamma=\left(L_{i}\right.$ : goto $\left.L_{j} ;\right)$. By the case assumption, we have $\left\langle L_{i}, n_{1}, n_{2}\right\rangle \sim M\left\langle L_{j}, n_{1}, n_{2}\right\rangle$, and we are required to show that the following sequent is derivable:

$$
\kappa(M) * l_{i} * p_{1}^{n_{1}} * p_{2}^{n_{2}} \vdash--l_{0} .
$$


By the induction hypothesis, we can derive

$$
\kappa(M) * l_{j} * p_{1}^{n_{1}} * p_{2}^{n_{2}} \vdash--l_{0} .
$$

By applying part (d) of Lemma 8.1, we obtain

$$
\kappa(M) *--l_{j} * p_{1}^{n_{1}} * p_{2}^{n_{2}} \vdash----l_{0} .
$$

Since --- $-l_{0} \vdash--l_{0}$ is derivable by part (b) of Lemma 8.1, we obtain by (Tr)

$$
\kappa(M) *--l_{j} * p_{1}^{n_{1}} * p_{2}^{n_{2}} \vdash--l_{0} .
$$

Now since $\left(-l_{j} *-l_{i}\right) * l_{i} \vdash--l_{j}$ is provable by part (c) of Lemma 8.1, we obtain by (Tr) and the derived rule $(*)$

$$
\kappa(M) *\left(-l_{j} *-l_{i}\right) * l_{i} * p_{1}^{n_{1}} * p_{2}^{n_{2}} \vdash--l_{0} .
$$

Since $\gamma \in M$ and $\kappa(\gamma)=\left(-l_{j} *-l_{i}\right)$, we have $\kappa(M) \vdash \kappa(M) *\left(-l_{j} *-l_{i}\right)$ provable by Lemma 8.2. Thus, we derive by (Tr) and ( $*$ ):

$$
\kappa(M) * l_{i} * p_{1}^{n_{1}} * p_{2}^{n_{2}} \vdash--l_{0},
$$

which completes the case.

Case $\gamma=\left(L_{i}: c_{1}++\right.$; goto $\left.L_{j} ;\right)$. By the case assumption, we have $\left\langle L_{i}, n_{1}, n_{2}\right\rangle \leadsto M$ $\left\langle L_{j}, n_{1}+1, n_{2}\right\rangle$, and we are required to show that the following sequent is derivable:

$$
\kappa(M) * l_{i} * p_{1}^{n_{1}} * p_{2}^{n_{2}} \vdash--l_{0} .
$$

By the induction hypothesis, we can derive

$$
\kappa(M) * l_{j} * p_{1}^{n_{1}+1} * p_{2}^{n_{2}} \vdash--l_{0} .
$$

By applying part (d) of Lemma 8.1, we obtain

$$
\kappa(M) *--\left(l_{j} * p_{1}\right) * p_{1}^{n_{1}} * p_{2}^{n_{2}} \vdash----l_{0} .
$$

Since $----l_{0} \vdash--l_{0}$ is derivable by part (b) of Lemma 8.1 , we obtain by ( $\mathrm{Tr}$ )

$$
\kappa(M) *--\left(l_{j} * p_{1}\right) * p_{1}^{n_{1}} * p_{2}^{n_{2}} \vdash--l_{0} .
$$

Now since $\left(-\left(l_{j} * p_{1}\right) *-l_{i}\right) * l_{i} \vdash--\left(l_{j} * p_{1}\right)$ is provable by part (c) of Lemma 8.1 , we obtain by $(\mathrm{Tr})$ and $(*)$

$$
\kappa(M) *\left(-\left(l_{j} * p_{1}\right) \rightarrow-l_{i}\right) * l_{i} * p_{1}^{n_{1}} * p_{2}^{n_{2}} \vdash--l_{0} .
$$

As $\gamma \in M$ and $\kappa(\gamma)=\left(-\left(l_{j} * p_{1}\right) *-l_{i}\right)$, we have $\kappa(M) \vdash \kappa(M) *\left(-\left(l_{j} * p_{1}\right) *-l_{i}\right)$ provable by Lemma 8.2. Thus, we derive by (Tr) and $(*)$

$$
\kappa(M) * l_{i} * p_{1}^{n_{1}} * p_{2}^{n_{2}} \vdash--l_{0},
$$

which completes the case.

Case $\gamma=\left(L_{i}: c_{1}--\right.$; goto $\left.L_{j} ;\right)$. By the case assumption, we have $\left\langle L_{i}, n_{1}+1, n_{2}\right\rangle \leadsto M$ $\left\langle L_{j}, n_{1}, n_{2}\right\rangle$, and we are required to show that the following sequent is derivable:

$$
\kappa(M) * l_{i} * p_{1}^{n_{1}+1} * p_{2}^{n_{2}} \vdash--l_{0} .
$$

By the induction hypothesis, we can derive

$$
\kappa(M) * l_{j} * p_{1}^{n_{1}} * p_{2}^{n_{2}} \vdash--l_{0}
$$

Using parts (b), (c), and (d) of Lemma 8.1 together with (Tr) and (-*) in a similar way to previous cases, we obtain

$$
\kappa(M) *\left(-l_{j} *-\left(l_{i} * p_{1}\right)\right) * l_{i} * p_{1}^{n_{1}+1} * p_{2}^{n_{2}} \vdash--l_{0} .
$$


Since $\gamma \in M$ and $\kappa(\gamma)=\left(-l_{j} *-\left(l_{i} * p_{1}\right)\right)$, we have $\kappa(M) \vdash \kappa(M) *\left(-l_{j} *-\left(l_{i} * p_{1}\right)\right)$ provable by Lemma 8.2. Thus, we derive by $(\mathrm{Tr})$ and $(*)$, as required:

$$
\kappa(M) * l_{i} * p_{1}^{n_{1}+1} * p_{2}^{n_{2}} \vdash--l_{0} .
$$

Case $\gamma=\left(L_{i}\right.$ : if $c_{1}=0$ goto $L_{j} ;$ ). By the case assumption, we have $\left\langle L_{i}, 0, n_{2}\right\rangle \sim M$ $\left\langle L_{j}, 0, n_{2}\right\rangle$, and must derive the following sequent:

$$
\kappa(M) * l_{i} * \mathrm{I} * p_{2}^{n_{2}} \vdash--l_{0} .
$$

First, by the induction hypothesis, we can derive

$$
\kappa(M) * l_{j} * \mathrm{I} * p_{2}^{n_{2}} \vdash--l_{0} .
$$

Next, note that by Lemma 7.2 , we have $\left\langle L_{-1}, 0, n_{2}\right\rangle \Downarrow_{M}$. Since the computation of $\left\langle L_{-1}, 0, n_{2}\right\rangle \sim_{M}^{*}\left\langle L_{0}, 0,0\right\rangle$ involves only decrement and goto instructions (see group (18) in Definition 7.1) by construction, the cases already considered above are sufficient to establish the present theorem for the configuration $\left\langle L_{-1}, 0, n_{2}\right\rangle$. Thus, we can also derive

$$
\kappa(M) * l_{-1} * \mathrm{I} * p_{2}^{n_{2}} \vdash--l_{0} .
$$

Thus, by applying part (e) of Lemma 8.1, we obtain from (23) and (24)

$$
\kappa(M) *--\left(l_{j} \vee l_{-1}\right) * \mathrm{I} * p_{2}^{n_{2}} \vdash----l_{0} .
$$

Since $----l_{0} \vdash--l_{0}$ is derivable by part (b) of Lemma 8.1, we obtain by (Tr)

$$
\kappa(M) *--\left(l_{j} \vee l_{-1}\right) * \mathrm{I} * p_{2}^{n_{2}} \vdash--l_{0} .
$$

Then, as $\left(-\left(l_{j} \vee l_{-1}\right) *-l_{i}\right) * l_{i} \vdash--\left(l_{j} \vee l_{-1}\right)$ is provable by part (c) of Lemma 8.1 , we obtain by $(\mathrm{Tr})$ and $(*)$

$$
\kappa(M) *\left(-\left(l_{j} \vee l_{-1}\right) *-l_{i}\right) * \mathrm{I} * l_{i} * p_{2}^{n_{2}} \vdash--l_{0} .
$$

Since $\gamma \in M$ and $\kappa(\gamma)=\left(-\left(l_{j} \vee l_{-1}\right) *-l_{i}\right)$, we have $\kappa(M) \vdash \kappa(M) *\left(-\left(l_{j} \vee l_{-1}\right) *-l_{i}\right)$ provable by Lemma 8.2. Thus, we derive by $(\mathrm{Tr})$ and $(*)$

$$
\kappa(M) * l_{i} * \mathrm{I} * p_{2}^{n_{2}} \vdash--l_{0},
$$

which completes the case, and the proof.

\section{FROM VALIDITY TO TERMINATING COMPUTATIONS}

In this section, our goal is to show that for each of the concrete models given in Section 2 , we have $\left\langle L_{i}, n_{1}, n_{2}\right\rangle \Downarrow_{M}$ whenever the following sequent $\mathcal{F}_{M,\left\langle L_{i}, n_{1}, n_{2}\right\rangle}$ is valid in this particular model

$$
\kappa(M) * l_{i} * p_{1}^{n_{1}} * p_{2}^{n_{2}} *\left(\mathrm{I} \wedge-l_{0}\right) \vdash b
$$

For the sake of perspicuity, we establish this property first for the simplest such model, the RAM-domain model from Section 2.5.

Then, we show how to extend our approach to any of the memory models from Section 2.

In particular, we extend our approach to the most general stack-and-heap models from Section 2.4, of which all the models in Sections 2.1-2.5 can be seen as special instances (see Remark 2.1).

Notice that, to provide undecidability, the set of locations $L$ must be infinite (cf. Sections 9.2 and 10).

The RAM-domain model case also suffices to cover the Petri net models from Section 2.7.

As for the finite multiset models of Section 2.6, the (un)desired undecidability property can be established even in the case of the multisets over a fixed finite set $L$ (see Section 9.5). 


\subsection{Valuation for the RAM-Domain Model}

Definition 9.1. Given a machine $M$, we introduce the following valuation $\rho_{0}$ for the RAM-domain model (D, $\left.\mathcal{D},\left\{e_{0}\right\}\right)$ of Section 2.5:

$$
\begin{array}{rlcl}
\rho_{0}\left(p_{1}\right) & = & \{\{6\},\{10\},\{14\},\{18\} \ldots,\} & =\{\{2(2 m+1)\} \mid m \geq 1\} \\
\rho_{0}\left(p_{2}\right) & =\quad\{\{12\},\{20\},\{28\},\{36\} \ldots\} & =\{\{4(2 m+1)\} \mid m \geq 1\} \\
\rho_{0}\left(l_{i}\right) & =\left\{\left\{2^{i+5} \cdot 3\right\},\left\{2^{i+5} \cdot 5\right\},\left\{2^{i+5} \cdot 7\right\}, \ldots\right\} & =\left\{\left\{2^{i+5} \cdot(2 m+1)\right\} \mid m \geq 1\right\},
\end{array}
$$

where $i \geq-2$, and

$$
\rho_{0}(b)=\bigcup_{\left\langle L_{i}, n_{1}, n_{2}\right\rangle \Downarrow_{M}} \llbracket l_{i} * p_{1}^{n_{1}} * p_{2}^{n_{2}} \rrbracket_{\rho_{0}},
$$

where $b$ is the distinguished propositional variable introduced in Definition 7.3. (The reason behind our choice for $\rho_{0}(b)$ is given in Section 7.1.)

Lemma 9.2. For any $d \in \mathcal{D}, n \in \mathbb{N}$ and $k \in\{1,2\}$, Definition 9.1 guarantees that $d \in \llbracket p_{k}^{n} \rrbracket_{\rho_{0}}$ if and only if $d$ consists of exactly $n$ distinct numbers of the form $2^{k} \cdot(2 m+1)$ (where $m \geq 1$ ).

Thus, $\llbracket l_{i} * p_{1}^{n_{1}} * p_{2}^{n_{2}} \rrbracket \rho_{\rho_{0}}$ is not empty, and each of the elements of $\llbracket l_{i} * p_{1}^{n_{1}} * p_{2}^{n_{2}} \rrbracket \rho_{\rho_{0}}$ uniquely determines the configuration $\left\langle L_{i}, n_{1}, n_{2}\right\rangle$.

Proof. The first part is by induction on $n$. In the case $n=0$, we have $\llbracket p_{k}^{0} \rrbracket_{\rho_{0}}=\llbracket \mathrm{I} \rrbracket_{\rho_{0}}=$ $\left\{e_{0}\right\}$, where $e_{0}$ is $\emptyset$. Thus, $d \in \llbracket p_{k}^{0} \rrbracket_{\rho_{0}}$ iff $d=\emptyset$ iff $d$ consists of zero numbers of the form $2^{k} \cdot(2 m+1)$.

In the case $n>0$, we have, using Lemma 3.8:

$$
\begin{aligned}
\llbracket p_{k}^{n} \rrbracket_{\rho_{0}} & =\llbracket p_{k} * p_{k}^{n-1} \rrbracket_{\rho_{0}} \\
& =\llbracket p_{k} \rrbracket_{\rho_{0}} \cdot \llbracket p_{k}^{n-1} \rrbracket_{\rho_{0}} \\
& =\left\{d^{\prime} \circ d^{\prime \prime} \mid d^{\prime} \in \rho_{0}\left(p_{k}\right), d^{\prime \prime} \in \llbracket p_{k}^{n-1} \rrbracket_{\rho_{0}}\right\} .
\end{aligned}
$$

Thus, $d \in \llbracket p_{k}^{n} \rrbracket_{\rho_{0}}$ iff it is the union of disjoint sets $d^{\prime}$ and $d^{\prime \prime}$ with $d^{\prime} \in \rho_{0}\left(p_{k}\right)$ and $d^{\prime \prime} \in \llbracket p_{k}^{n-1} \rrbracket_{\rho_{0}}$. By induction hypothesis, $d^{\prime \prime} \in \llbracket p_{k}^{n-1} \rrbracket_{\rho_{0}}$ iff $d^{\prime \prime}$ consists of exactly $n-1$ distinct numbers of the form $2^{k} \cdot(2 m+1)$, and by construction it holds that $d^{\prime} \in \rho_{0}\left(p_{k}\right)$ iff $d^{\prime}=\left\{2^{k} \cdot\left(2 m^{\prime}+1\right)\right\}$ for some $m^{\prime}$. Since $d^{\prime}$ and $d^{\prime \prime}$ must be disjoint, we have $d \in \llbracket p_{k}^{n} \rrbracket_{\rho_{0}}$ iff $d$ consists of exactly $n$ numbers of the form $2^{k} \cdot(2 m+1)$, as required.

For the second part of the lemma, we have, using Lemma 3.8:

$$
\begin{aligned}
\llbracket l_{i} * p_{1}^{n_{1}} * p_{2}^{n_{2}} \rrbracket_{\rho_{0}} & =\llbracket l_{i} \rrbracket_{\rho_{0}} \cdot \llbracket p_{1}^{n_{1}} \rrbracket \rho_{\rho_{0}} \cdot \llbracket p_{2}^{n_{2}} \rrbracket \\
& =\left\{d_{1} \circ d_{2} \circ d_{3} \mid d_{1} \in \rho_{0}\left(l_{i}\right), d_{2} \in \llbracket p_{1}^{n_{1}} \rrbracket \rho_{\rho_{0}}, d_{3} \in \llbracket p_{2}^{n_{2}} \rrbracket_{\rho_{0}}\right\} .
\end{aligned}
$$

Thus, $d \in \llbracket l_{i} * p_{1}^{n_{1}} * p_{2}^{n_{2}} \rrbracket_{\rho_{0}}$ iff it is the union of disjoint sets $d_{1}, d_{2}$ and $d_{3}$ with $d_{1} \in \rho_{0}\left(l_{i}\right)$, $d_{2} \in \llbracket p_{1}^{n_{1}} \rrbracket_{\rho_{0}}$ and $d_{3} \in \llbracket p_{2}^{n_{2}} \rrbracket_{\rho_{0}}$. By the first part of the lemma, $d_{2}$ and $d_{3}$ consist of $n_{1}$ distinct numbers of form $2(2 m+1)$ and $n_{2}$ distinct numbers of the form $4(2 m+1)$, respectively, whereas by construction $d_{1}=\left\{2^{i+5} \cdot\left(2 m^{\prime}+1\right)\right\}$ for some $m^{\prime} \geq 1$ and $i \geq-2$.

To see that the decomposition of $d$ into $d_{1} \circ d_{2} \circ d_{3}$ exists and is unique, we must show that $d_{1}, d_{2}, d_{3}$ are nonoverlapping. To see this, suppose that $2^{i} \cdot(2 m+1)=2^{j}$. $\left(2 m^{\prime}+1\right)$ where $i \neq j$, and assume without loss of generality that $j>i$. Then, by simple manipulation, we have $2 m=2^{j-i+1} m^{\prime}+2^{j-i}-1$, but then $2 m$ is even while $2^{j-i+1} m^{\prime}+2^{j-i}-1$ is odd, contradiction. Thus, $\llbracket l_{i} * p_{1}^{n_{1}} * p_{2}^{n_{2}} \rrbracket_{\rho_{0}}$ is not empty, and each of its elements uniquely determines $L_{i}, n_{1}$ and $n_{2}$, and hence the configuration $\left\langle L_{i}, n_{1}, n_{2}\right\rangle$. 


\subsection{Do We Need Infinite Valuations?}

Our choice of $\rho_{0}\left(p_{1}\right)$ and $\rho_{0}\left(p_{2}\right)$ to have infinitely many disjoint elements is dictated by peculiarities of composition $\circ$ in the heap model. Moreover, for any finite choice of $\rho_{0}\left(p_{k}\right)$, we can show that for all sufficiently large $n$,

$$
\llbracket p_{k}^{n} \rrbracket_{\rho_{0}}=\emptyset,
$$

which obstructs us in uniquely representing the contents $n$ of the Minsky machine counter $c_{k}$ by the formula $p_{k}^{n}$. We discuss the impact upon decidability of a restriction to finite valuations in Section 10.

\subsection{Formal Details}

Now we prove the basic lemma.

Lemma 9.3. $e_{0} \models_{\rho_{0}} \kappa(M)$ for any machine $M$.

Proof. Writing $M=\left\{\gamma_{1}, \ldots, \gamma_{t}\right\}$, we have by Definition 3.7:

$$
\begin{aligned}
e_{0} \models_{\rho_{0}} \kappa(M) & \Leftrightarrow e_{0} \models_{\rho_{0}} \mathrm{I} \wedge \bigwedge_{i=1}^{t} \kappa\left(\gamma_{i}\right) \\
& \Leftrightarrow e_{0} \in\left\{e_{0}\right\} \text { and } \forall i \in\{1, \ldots, t\} . e_{0} \models_{\rho_{0}} \kappa\left(\gamma_{i}\right)
\end{aligned}
$$

Thus, it suffices to show that $e_{0} \models_{\rho_{0}} \kappa(\gamma)$ for any instruction $\gamma$. Recalling that $-A=_{\text {def }}(A * b)$, we shall make use of the fact that, for any $d \in \mathcal{D}$ and any formula A,

$$
d \models_{\rho_{0}}-A \Leftrightarrow \forall d^{\prime} .\left(d, d^{\prime} \text { disjoint and } d^{\prime} \models_{\rho_{0}} A\right) \text { implies } d \circ d^{\prime} \in \rho_{0}(b) \text {. }
$$

We present the cases for a goto instruction, and for increment, decrement and zero-test instructions with counter variable $k=1$; the cases where $k=2$ are similar.

Case $\gamma=\left(L_{i}\right.$ : goto $L_{j}$;). We have $\kappa(\gamma)=\left(-l_{j} *-l_{i}\right)$, so must show $e_{0} \models_{\rho_{0}}-l_{j} *-l_{i}$. Using the fact that $e_{0} \circ d=d$, this amounts to showing, for any $d \in \mathcal{D}$,

$$
d \models \rho_{0}-l_{j} \text { implies } d \models_{\rho_{0}}-l_{i} .
$$

To show this implication, suppose $d \models_{\rho_{0}}-l_{j}$. Since $d \in \mathcal{D}$ is a finite set whereas $\rho_{0}\left(l_{j}\right)$ contains infinitely many disjoint sets by construction, there must be some (in fact, infinitely many) $d_{j} \in \rho_{0}\left(l_{j}\right)$ such that $d$ and $d^{\prime}$ are disjoint. Thus, using equivalence (25) and Lemma 3.8, we have:

$$
\begin{aligned}
d \circ d_{j} & \in \rho_{0}(b) \\
& =\bigcup_{\left\langle L_{i}, n_{1}, n_{2}\right\rangle \Downarrow_{M}} \llbracket l_{i} * p_{1}^{n_{1}} * p_{2}^{n_{2}} \rrbracket_{\rho_{0}} \\
& =\bigcup_{\left\langle L_{i}, n_{1}, n_{2}\right\rangle \Downarrow_{M}} \rho_{0}\left(l_{i}\right) \cdot \llbracket p_{1}^{n_{1}} * p_{2}^{n_{2}} \rrbracket \rho_{0} .
\end{aligned}
$$

By construction of $\rho_{0}$ and using the fact that $d_{j} \in \rho_{0}\left(l_{j}\right)$, we must have $d \in \llbracket p_{1}^{n_{1}} * p_{2}^{n_{2}} \rrbracket_{\rho_{0}}$ for some $n_{1}, n_{2}$. Moreover, by Lemma $9.2, d \circ d_{j}$ uniquely determines $\left\langle L_{j}, n_{1}, n_{2}\right\rangle$, so we must have $\left\langle L_{j}, n_{1}, n_{2}\right\rangle \Downarrow_{M}$. Since $\left\langle L_{i}, n_{1}, n_{2}\right\rangle \sim_{M}\left\langle L_{j}, n_{1}, n_{2}\right\rangle$ by applying the goto instruction $\gamma$, we then have $\left\langle L_{i}, n_{1}, n_{2}\right\rangle \Downarrow_{M}$.

Now to see that $d \models \rho_{0}-l_{i}$, let $d^{\prime} \in \mathcal{D}$ be disjoint from $d$ with $d^{\prime} \in \rho_{0}\left(l_{i}\right)$. By (25), it suffices to show that $d \circ d^{\prime} \in \rho_{0}(b)$. Since $d \in \llbracket p_{1}^{n_{1}} * p_{2}^{n_{2}} \rrbracket \rho_{0}$, we have $d \circ d^{\prime} \in \llbracket l_{i} * p_{1}^{n_{1}} * p_{2}^{n_{2}} \rrbracket \rho_{0}$ where $\left\langle L_{i}, n_{1}, n_{2}\right\rangle \Downarrow_{M}$, and thus $d \circ d^{\prime} \in \rho_{0}(b)$ as required.

Case $\gamma=\left(L_{i}\right.$ : $c_{1}++$; goto $\left.L_{j} ;\right)$. We have $\kappa(\gamma)=\left(-\left(l_{j} * p_{1}\right) *-l_{i}\right)$. As in the previous case, to show $e_{0}=\rho_{0} \kappa(\gamma)$, we must show that, for any $d \in \mathcal{D}$,

$$
d \models_{\rho_{0}}-\left(l_{j} * p_{1}\right) \text { implies } d \models_{\rho_{0}}-l_{i} \text {. }
$$


Suppose $d \models_{\rho_{0}}-\left(l_{j} * p_{1}\right)$. Since $\rho_{0}\left(l_{j}\right)$ and $\rho_{0}\left(p_{1}\right)$ each contain infinitely many disjoint sets, we can find $d_{j} \in \rho_{0}\left(l_{j}\right)$ and $d_{1} \in \rho_{0}\left(p_{1}\right)$ such that $d, d_{j}$, and $d_{1}$ are disjoint. Thus, $d_{j} \circ d_{1} \models_{\rho_{0}} l_{j} * p_{1}$. Using the equivalence (25) and Lemma 3.8, we have:

$$
\begin{aligned}
d \circ d_{j} \circ d_{1} & \in \rho_{0}(b) \\
& =\bigcup_{\left\langle L_{i}, n_{1}, n_{2}\right\rangle \Downarrow_{M}} \rho_{0}\left(l_{i}\right) \cdot \llbracket p_{1}^{n_{1}} * p_{2}^{n_{2}} \rrbracket_{\rho_{0}} .
\end{aligned}
$$

By construction of $\rho_{0}$ and using the fact that $d_{j} \in \rho_{0}\left(l_{j}\right)$, we must have $d \in \llbracket p_{1}^{n_{1}} * p_{2}^{n_{2}} \rrbracket \rho_{0}$ for some $n_{1}, n_{2}$. Moreover, by Lemma 9.2, and using the fact that $d_{1} \in \rho_{0}\left(p_{1}\right)$, the set $d \circ d_{j} \circ d_{1}$ uniquely determines $\left\langle L_{j}, n_{1}+1, n_{2}\right\rangle$, whence $\left\langle L_{j}, n_{1}+1, n_{2}\right\rangle \Downarrow_{M}$. Since $\left\langle L_{i}, n_{1}, n_{2}\right\rangle \sim_{M}\left\langle L_{j}, n_{1}+1, n_{2}\right\rangle$ via the increment instruction $\gamma$, we have $\left\langle L_{i}, n_{1}, n_{2}\right\rangle \Downarrow_{M}$.

Now, to see that $d \models \rho_{\rho_{0}}-l_{i}$, let $d^{\prime} \in \mathcal{D}$ be disjoint from $d$ with $d^{\prime} \in \rho_{0}\left(l_{i}\right)$. By (25), it suffices to show that $d \circ d^{\prime} \in \rho_{0}(b)$. Since $d \in \llbracket p_{1}^{n_{1}} * p_{2}^{n_{2}} \rrbracket \rho_{0}$, we have $d \circ d^{\prime} \in \llbracket l_{i} * p_{1}^{n_{1}} * p_{2}^{n_{2}} \rrbracket \rho_{0}$ where $\left\langle L_{i}, n_{1}, n_{2}\right\rangle \Downarrow_{M}$, and thus $d \circ d^{\prime} \in \rho_{0}(b)$ as required.

Case $\gamma=\left(L_{i}\right.$ : $c_{1}--$; goto $\left.L_{j} ;\right)$. We have $\kappa(\gamma)=\left(-l_{j} *-\left(l_{i} * p_{1}\right)\right)$. To show $e_{0}=_{\rho_{0}} \kappa(\gamma)$, we must show that, for any $d \in \mathcal{D}$,

$$
d \models_{\rho_{0}}-l_{j} \text { implies } d=_{\rho_{0}}-\left(l_{i} * p_{1}\right) \text {. }
$$

Now suppose $d=_{\rho_{0}}-l_{j}$. As in the previous cases, we can find $d_{j} \in \rho_{0}\left(l_{j}\right)$ such that $d_{j}$ and $d$ are disjoint. Thus, using equivalence (25), we have:

$$
\begin{aligned}
d \circ d_{j} & \in \rho_{0}(b) \\
& =\bigcup_{\left\langle L_{i}, n_{1}, n_{2}\right\rangle \Downarrow_{M}} \rho_{0}\left(l_{i}\right) \cdot \llbracket p_{1}^{n_{1}} * p_{2}^{n_{2}} \rrbracket_{\rho_{0}} .
\end{aligned}
$$

By the same argument as in previous cases, this implies that there exist $n_{1}$ and $n_{2}$ such that $d \in \llbracket p_{1}^{n_{1}} * p_{2}^{n_{2}} \rrbracket_{\rho_{0}}$, and $\left\langle L_{j}, n_{1}, n_{2}\right\rangle \Downarrow_{M}$. Since $\left\langle L_{i}, n_{1}+1, n_{2}\right\rangle \sim_{M}\left\langle L_{j}, n_{1}, n_{2}\right\rangle$ via the decrement instruction $\gamma$, we have $\left\langle L_{i}, n_{1}+1, n_{2}\right\rangle \Downarrow_{M}$.

Now let $d^{\prime} \in \mathcal{D}$ be disjoint from $d$ with $d^{\prime} \models_{\rho_{0}} l_{i} * p_{1}$. By (25), it suffices to show that $d \circ d^{\prime} \in \rho_{0}(b)$. First, we have $d^{\prime}=d_{i} \circ d_{1}$ where $d_{i} \in \rho_{0}\left(l_{i}\right)$ and $d_{1} \in \rho_{0}\left(p_{1}\right)$. Since $d \in \llbracket p_{1}^{n_{1}} * p_{2}^{n_{2}} \rrbracket_{\rho_{0}}$, we have $d \circ d_{i} \circ d_{1} \in \llbracket l_{i} * p_{1}^{n_{1}+1} * p_{2}^{n_{2}} \rrbracket_{\rho_{0}}$ where $\left\langle L_{i}, n_{1}+1, n_{2}\right\rangle \Downarrow_{M}$. Thus, $d \circ d_{i} \circ d_{1}=d \circ d^{\prime} \in \rho_{0}(b)$ as required.

Case $\gamma=\left(L_{i}:\right.$ if $c_{1}=0$ goto $\left.L_{j} ;\right)$. In this case, we have $\kappa(\gamma)=\left(-\left(l_{j} \vee l_{-1}\right) *-l_{i}\right)$. To show $e_{0} \models_{\rho_{0}} \kappa(\gamma)$, we must show that, for any $d \in \mathcal{D}$,

$$
d \models_{\rho_{0}}-\left(l_{j} \vee l_{-1}\right) \text { implies } d \models_{\rho_{0}}-l_{i} \text {. }
$$

Now suppose that $d \models_{\rho_{0}}-\left(l_{j} \vee l_{-1}\right)$. As in previous cases, we can find $d_{j} \in \rho_{0}\left(l_{j}\right)$ and $d_{-1} \in \rho_{0}\left(l_{-1}\right)$ such that $d, d_{j}$, and $d_{-1}$ are all disjoint. Note that both $d_{j} \models \rho_{0} l_{j} \vee l_{-1}$ and $d_{-1} \models_{\rho_{0}} l_{j} \vee l_{-1}$. Thus, using equivalence (25), we have:

$$
\begin{aligned}
d \circ d_{j}, d \circ d_{-1} & \in \rho_{0}(b) \\
& =\bigcup_{\left\langle L_{i}, n_{1}, n_{2}\right\rangle \Downarrow_{M}} \rho_{0}\left(l_{i}\right) \cdot \llbracket p_{1}^{n_{1}} * p_{2}^{n_{2}} \rrbracket_{\rho_{0}} .
\end{aligned}
$$

By the same argument as in previous cases, there are $n_{1}$ and $n_{2}$ such that $d \in \llbracket p_{1}^{n_{1}} * p_{2}^{n_{2}} \rrbracket_{\rho_{0}}$, and we have both $\left\langle L_{j}, n_{1}, n_{2}\right\rangle \Downarrow_{M}$ and $\left\langle L_{-1}, n_{1}, n_{2}\right\rangle \Downarrow_{M}$. However, by Lemma $7.2,\left\langle L_{-1}, n_{1}, n_{2}\right\rangle \Downarrow_{M}$ implies $n_{1}=0$. Thus, $\left\langle L_{i}, n_{1}, n_{2}\right\rangle \sim_{M}\left\langle L_{j}, n_{1}, n_{2}\right\rangle \Downarrow_{M}$ by applying the zero-test instruction $\gamma$, whence $\left\langle L_{i}, n_{1}, n_{2}\right\rangle \Downarrow_{M}$. The argument that $d=\rho_{0}-l_{i}$ is then exactly as in the goto and increment instruction cases. This completes the case, and the proof.

Lemma 9.4. For any machine $M$ we have $e_{0} \models_{\rho_{0}} \mathrm{I} \wedge-l_{0}$. 
Proof. We trivially have $e_{0} \models{ }_{\rho_{0}}$ I. To show $e_{0} \models_{\rho_{0}}-l_{0}$, taking into account that $e_{0} \circ x=x$, we must show that $x \models_{\rho_{0}} l_{0}$ implies $x \models_{\rho_{0}} b$, that is, that $\rho_{0}\left(l_{0}\right) \subseteq \rho_{0}(b)$. This is immediate by construction of $\rho_{0}(b)$, since $\left\langle L_{0}, 0,0\right\rangle \Downarrow_{M}$ by definition and $\rho_{0}\left(l_{0}\right)=\llbracket l_{0} * \mathrm{I} * \mathrm{I} \rrbracket_{\rho_{0}}=\llbracket l_{0} * p_{1}^{0} * p_{2}^{0} \rrbracket_{\rho_{0}}$.

Theorem 9.5. For any machine $M$, if the sequent

$$
\left(\kappa(M) * l_{i} * p_{1}^{n_{1}} * p_{2}^{n_{2}} *\left(\mathrm{I} \wedge-l_{0}\right)\right) \vdash b
$$

is valid in the RAM-domain model $\left(\mathcal{D}, \circ,\left\{e_{0}\right\}\right)$, then $\left\langle L_{i}, n_{1}, n_{2}\right\rangle \Downarrow_{M}$.

Proof. By the definition of validity and using Lemma 3.8, we have:

$$
\begin{aligned}
\llbracket \kappa(M) * l_{i} * p_{1}^{n_{1}} * p_{2}^{n_{2}} *\left(\mathrm{I} \wedge-l_{0}\right) \rrbracket \rho_{0} & \subseteq \llbracket b \rrbracket_{\rho_{0}} \\
\text { i.e., } \llbracket \kappa(M) \rrbracket_{\rho_{0}} \cdot \llbracket l_{i} * p_{1}^{n_{1}} * p_{2}^{n_{2}} \rrbracket_{\rho_{0}} \cdot \llbracket \mathrm{I} \wedge-l_{0} \rrbracket \rho_{0} & \subseteq \rho_{0}(b) .
\end{aligned}
$$

Since $e_{0} \in \llbracket \kappa(M) \rrbracket_{\rho_{0}}$ by Lemma 9.3 and $e_{0} \in \llbracket \mathrm{I} \wedge-l_{0} \rrbracket_{\rho_{0}}$. by Lemma 9.4 we have in particular:

$$
\llbracket l_{i} * p_{1}^{n_{1}} * p_{2}^{n_{2}} \rrbracket \rho_{0} \subseteq \rho_{0}(b) .
$$

By Lemma 9.2, each of the elements of the set $\llbracket l_{i} * p_{1}^{n_{1}} * p_{2}^{n_{2}} \rrbracket_{\rho_{0}}$ uniquely determines the configuration $\left\langle L_{i}, n_{1}, n_{2}\right\rangle$, whence our construction of $\rho_{0}(b)$ yields $\left\langle L_{i}, n_{1}, n_{2}\right\rangle \Downarrow_{M}$.

\subsection{The General Case of Stack-and-Heap-with-Permission Models}

Having established as Theorem 9.5 that validity of a sequent of the form $\mathcal{F}_{M, C}$ in the basic RAM-domain model implies termination of machine $M$ from configuration $C$, we now extend this result to the most sophisticated stack-and-heap-with-permission models from Section 2.4. As per Remark 2.1, all the models from Sections 2.1-2.5 can be seen as special instances of such models by taking appropriate locations $L$, record values $R V$, stacks $S$ and permission algebra $(P, \bullet, \mathbb{1})$. Without loss of generality, we consider the infinite set of locations $L$ to be given by $\mathbb{N}$. We write $o^{\prime}$ for the composition in a stack-and-heap-with-permission model and '. for its extension to sets to disambiguate from the corresponding operations $\circ$ and · of the RAM-domain model.

Definition 9.6. Let $\left(S \times H, \circ^{\prime}, E\right)$ be a stack-and-heap model from Section 2.4, where $S$ is a set of stacks and $H$ is a set of heaps-with-permissions from locations $L=\mathbb{N}$ to pairs of record values and permissions in $R V \times P$, where $(P, \bullet, \mathbb{1})$ is a permission algebra. (Recall that $\mathbb{1} \bullet \pi$ is undefined for all $\pi \in P$.) We abuse notation slightly by writing the unit $e_{0}$ of the RAM-domain model for the heap with empty domain. Thus, $E=\left\{\left\langle s, e_{0}\right\rangle \mid s \in S\right\}$.

Based on our valuation $\rho_{0}$ for the RAM-domain model and a given machine $M$ in Definition 9.1, we introduce a valuation $\rho_{1}$ for $\left(S \times H, \circ^{\prime}, E\right)$ as follows. First, we fix an arbitrary stack $s_{0} \in S$, and for each finite set $d \subseteq \mathbb{N}$ we define the set $[d] \subseteq S \times H$ by:

$$
[d]=\left\{\left\langle s_{0}, h\right\rangle \mid \operatorname{domain}(h)=d \text { and } \forall \ell \in d . h(\ell)=\left\langle_{-}, \mathbb{1}\right\rangle\right\} .
$$

where $h(\ell)=\left\langle \_, \mathbb{1}\right\rangle$ means that $h(\ell)=\langle s, \mathbb{1}\rangle$ for some $s \in S$. Then, for any atomic proposition $p$, we define its valuation by:

$$
\rho_{1}(p)=\bigcup_{d \in \rho_{0}(p)}[d]
$$

Lemma 9.7. For any stack-and-heap model $\left(S \times H, \circ^{\prime}, E\right)$ and any atomic propositions $p$ and $q$, we have the following identity:

$$
\llbracket p * q \rrbracket_{\rho_{1}}=\bigcup_{d \in \llbracket p * q \rrbracket_{\rho_{0}}}[d] .
$$


Proof. First, we show that for any $d_{1} \in \rho_{0}(p), d_{2} \in \rho_{0}(q)$,

$$
\left[d_{1} \circ d_{2}\right]=\left[d_{1}\right] \cdot^{\prime}\left[d_{2}\right] \text {. }
$$

For disjoint $d_{1}$ and $d_{2}$, this is given by construction, since

$$
\begin{aligned}
{\left[d_{1}\right] \circ^{\prime}\left[d_{2}\right] } & =\left\{\left\langle s_{0}, h_{1}\right\rangle \circ^{\prime}\left\langle s_{0}, h_{2}\right\rangle \mid\left\langle s_{0}, h_{1}\right\rangle \in\left[d_{1}\right],\left\langle s_{0}, h_{2}\right\rangle \in\left[d_{2}\right]\right\} \\
& =\left\{\left\langle s_{0}, h_{1}\right\rangle \circ^{\prime}\left\langle s_{0}, h_{2}\right\rangle \mid \operatorname{domain}\left(h_{1}\right)=\left[d_{1}\right], \operatorname{domain}\left(h_{2}\right)=\left[d_{2}\right],\right. \\
& \quad \forall \ell \in d_{1} . h_{1}(\ell)=\left\langle\left\langle_{-}, \mathbb{1}\right\rangle, \forall \ell \in d_{2} . h_{2}(\ell)=\left\langle_{-}, \mathbb{1}\right\rangle\right\} \\
& =\left\{\left\langle s_{0}, h\right\rangle \mid \operatorname{domain}(h)=d_{1} \circ d_{2}, \forall \ell \in d_{1} \circ d_{2} . h(\ell)=\left\langle{ }_{-}, \mathbb{1}\right\rangle\right\} \\
& =\left[d_{1} \circ d_{2}\right] .
\end{aligned}
$$

For overlapping $d_{1}$ and $d_{2}$, we have $d_{1} \circ d_{2}$ undefined and thus $\left[d_{1} \circ d_{2}\right]=\emptyset$. We show that $\left[d_{1}\right] .^{\prime}\left[d_{2}\right]=\emptyset$. Assume for contradiction that $\left\langle s_{0}, h_{1}\right\rangle \circ^{\prime}\left\langle s_{0}, h_{2}\right\rangle$ is defined for some $\left\langle s_{0}, h_{1}\right\rangle \in\left[d_{1}\right]$ and $\left\langle s_{0}, h_{2}\right\rangle \in\left[d_{2}\right]$. By construction of $\left[d_{1}\right]$ and $\left[d_{2}\right]$, and since $d_{1}$, $d_{2}$ are overlapping, this implies $h_{1}(\ell)=h_{2}(\ell)=\left\langle_{-}, \mathbb{1}\right\rangle$ for some $\ell \in d_{1} \cap d_{2}$. But then since $\left\langle s_{0}, h_{1}\right\rangle \circ^{\prime}\left\langle s_{0}, h_{2}\right\rangle$ is defined, we must have $\mathbb{1} \bullet \mathbb{1}$ defined (because in heap-withpermissions models (cf. Section 2.3) we add the permissions when composing compatible heaps), which is a contradiction. Thus, $\left[d_{1}\right] \cdot^{\prime}\left[d_{2}\right]$ is empty when $d_{1} \circ d_{2}$ is undefined.

Now, using Lemma 3.8, we have as required:

$$
\begin{aligned}
& \llbracket p * q \rrbracket_{\rho_{1}}=\llbracket p \rrbracket_{\rho_{1}} \cdot^{\prime} \llbracket q \rrbracket_{\rho_{1}} \\
& =\left\{\left\langle s_{0}, h_{1}\right\rangle \circ^{\prime}\left\langle s_{0}, h_{2}\right\rangle \mid\left\langle s_{0}, h_{1}\right\rangle \in \rho_{1}(p),\left\langle s_{0}, h_{2}\right\rangle \in \rho_{1}(q)\right\} \\
& =\left\{\left\langle s_{0}, h_{1}\right\rangle \circ^{\prime}\left\langle s_{0}, h_{2}\right\rangle \mid\left\langle s_{0}, h_{1}\right\rangle \in \bigcup_{d \in \rho_{0}(p)}[d],\left\langle s_{0}, h_{2}\right\rangle \in \bigcup_{d \in \rho_{0}(q)}[d]\right\} \\
& =\bigcup_{d_{1} \in \rho_{0}(p), d_{2} \in \rho_{0}(q)}\left\{\left\langle s_{0}, h_{1}\right\rangle \circ^{\prime}\left\langle s_{0}, h_{2}\right\rangle \mid\left\langle s_{0}, h_{1}\right\rangle \in\left[d_{1}\right],\left\langle s_{0}, h_{2}\right\rangle \in\left[d_{2}\right]\right\} \\
& =\bigcup_{d_{1} \in \rho_{0}(p), d_{2} \in \rho_{0}(q)}\left[d_{1}\right] \bullet^{\prime}\left[d_{2}\right] \\
& =\bigcup_{d_{1} \in \rho_{0}(p), d_{2} \in \rho_{0}(q)}\left[d_{1} \circ d_{2}\right] \\
& =\bigcup_{d \in\left\{d_{1} \circ d_{2} \mid d_{1} \in \rho_{0}(p), d_{2} \in \rho_{0}(q)\right\}}[d]
\end{aligned}
$$

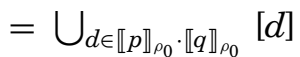

$$
\begin{aligned}
& =\bigcup_{d \in \llbracket p * q \rrbracket_{\rho_{0}}}[d] \text {. }
\end{aligned}
$$

Lemma 9.8. For any stack-and-heap model $\left(S \times H, o^{\prime}, E\right)$ and any formula A of the form $l_{i}, l_{i} * p_{k}$, or $l_{i} \vee l_{j}$, we have the equivalence

$$
\llbracket A \rrbracket_{\rho_{1}} \cdot\left\{\left\langle s_{0}, h\right\rangle\right\} \subseteq \llbracket b \rrbracket_{\rho_{1}} \Leftrightarrow\left\langle s_{0}, h\right\rangle \in[d] \text { and } \llbracket A \rrbracket_{\rho_{0}} \cdot\{d\} \subseteq \llbracket b \rrbracket_{\rho_{0}},
$$

where $d=\operatorname{domain}(h)$.

Proof. First, note that by distinguishing cases on $A$, and using Lemma 9.7 in the case $A=l_{i} * p_{k}$, we easily have that

$$
\llbracket A \rrbracket_{\rho_{1}}=\bigcup_{d \in \llbracket A \rrbracket_{\rho_{0}}}[d] .
$$

We show each of the required implications separately.

$(\Rightarrow)$ Suppose that $\llbracket A \rrbracket_{\rho_{1}} \cdot\left\{\left\langle s_{0}, h\right\rangle\right\} \subseteq \llbracket b \rrbracket_{\rho_{1}}$. That is, we have:

$$
\left\{\left\langle s^{\prime}, h^{\prime}\right\rangle \circ^{\prime}\left\langle s_{0}, h\right\rangle \mid\left\langle s^{\prime}, h^{\prime}\right\rangle \models \rho_{1} A\right\} \subseteq \bigcup_{d \in \rho_{0}(b)}[d] .
$$

Now let $d=\operatorname{domain}(h)$, and let $x \in \llbracket A \rrbracket_{\rho_{0}} \cdot\{d\}$. Thus, $x=d \circ d^{\prime}$, where $d$ and $d^{\prime}$ are disjoint and $d^{\prime} \models \rho_{\rho_{0}} A$. We require to show that $d \circ d^{\prime} \in \llbracket b \rrbracket_{\rho_{0}}$ and $\left\langle s_{0}, h\right\rangle \in[d]$. 
Let $\left\langle s_{0}, h^{\prime}\right\rangle \in\left[d^{\prime}\right]$. Since $d^{\prime} \in \llbracket A \rrbracket_{\rho_{0}}$, we have by $(27)\left\langle s_{0}, h^{\prime}\right\rangle \in \llbracket A \rrbracket_{\rho_{1}}$, that is, $\left\langle s_{0}, h^{\prime}\right\rangle \models_{\rho_{1}} A$. Since $d$ and $d^{\prime}$ are disjoint, $\left\langle s_{0}, h\right\rangle \circ^{\prime}\left\langle s_{0}, h^{\prime}\right\rangle$ is defined and, by (28),

$$
\left\langle s_{0}, h\right\rangle \circ^{\prime}\left\langle s_{0}, h^{\prime}\right\rangle \in \bigcup_{d \in \rho_{0}(b)}[d] .
$$

That is, $\left\langle s_{0}, h\right\rangle \circ^{\prime}\left\langle s_{0}, h^{\prime}\right\rangle \in\left[d^{\prime \prime}\right]$ for some $d^{\prime \prime} \in \rho_{0}(b)$. Since the domain of $\left\langle s_{0}, h\right\rangle \circ^{\prime}\left\langle s_{0}, h^{\prime}\right\rangle$ is $d \circ d^{\prime}$ by construction, we must have $d^{\prime \prime}=d \circ d^{\prime}$. Thus, $d \circ d^{\prime} \in \rho_{0}(b)=\llbracket b \rrbracket_{\rho_{0}}$ as required. Furthermore, $\left\langle s_{0}, h\right\rangle \in[d]$ because $d$ and $d^{\prime}$ are disjoint, and thus $h$ and $h^{\prime}$ do not overlap, but $\left\langle s_{0}, h\right\rangle \circ^{\prime}\left\langle s_{0}, h^{\prime}\right\rangle \in\left[d \circ d^{\prime}\right]$, which means that $h(\ell)=\left\langle_{-}, \mathbb{1}\right\rangle$ for all $\ell \in d$. This completes the "only if" direction.

$(\Leftarrow)$ Let $d=\operatorname{domain}(h)$, let $\left\langle s_{0}, h\right\rangle \in[d]$ and suppose $\llbracket A \rrbracket_{\rho_{0}} \cdot\{d\} \subseteq \llbracket b \rrbracket_{\rho_{0}}$. The latter inclusion means that:

$$
\left\{d^{\prime} \circ d \mid d^{\prime} \models \rho_{0} A\right\} \subseteq \rho_{0}(b) .
$$

Now suppose that $x \in \llbracket A \rrbracket_{\rho_{1}} \cdot^{\prime}\left\{\left\langle s_{0}, h\right\rangle\right\}$. Thus, $x=\left\langle s^{\prime}, h^{\prime}\right\rangle \circ^{\prime}\left\langle s_{0}, h\right\rangle$ (which implies $s^{\prime}=s_{0}$ ), where $\left\langle s^{\prime}, h^{\prime}\right\rangle \models_{\rho_{1}} A$. We require to show that $\left\langle s_{0}, h^{\prime}\right\rangle \circ^{\prime}\left\langle s_{0}, h\right\rangle \in \llbracket b \rrbracket_{\rho_{1}}$.

Since $\left\langle s_{0}, h^{\prime}\right\rangle \in \llbracket A \rrbracket_{\rho_{1}}$, we have by (27) some $d^{\prime}$ such that $d^{\prime} \in \llbracket A \rrbracket_{\rho_{0}}$, that is, $d^{\prime} \models_{\rho_{0}} A$, and $\left\langle s_{0}, h^{\prime}\right\rangle \in\left[d^{\prime}\right]$ (which implies domain $\left(h^{\prime}\right)=d^{\prime}$ ). Because $\left\langle s_{0}, h\right\rangle \in[d]$ and $\left\langle s^{\prime}, h^{\prime}\right\rangle \in\left[d^{\prime}\right]$, we have $h(\ell)=\left\langle_{-}, \mathbb{1}\right\rangle$ for all $\ell \in d$ and $h^{\prime}\left(\ell^{\prime}\right)=\left\langle_{-}, \mathbb{1}\right\rangle$ for all $\ell^{\prime} \in d^{\prime}$. As $\left\langle s_{0}, h^{\prime}\right\rangle \circ^{\prime}\left\langle s_{0}, h\right\rangle$ is defined by assumption, it follows that $h$ and $h^{\prime}$ cannot overlap, and so $d$ and $d^{\prime}$ are disjoint. Thus, by (29), $d^{\prime} \circ d \in \rho_{0}(b)$. We easily have $\left\langle s_{0}, h^{\prime}\right\rangle \circ^{\prime}\left\langle s_{0}, h\right\rangle \in\left[d^{\prime} \circ d\right]$, and so

$$
\left\langle s_{0}, h^{\prime}\right\rangle \circ^{\prime}\left\langle s_{0}, h\right\rangle \in \bigcup_{d \in \rho_{0}(b)}[d]=\rho_{1}(b)=\llbracket b \rrbracket_{\rho_{1}},
$$

as required. This completes the "if" direction, and the proof.

LEMMA 9.9. $\left\langle s_{0}, e_{0}\right\rangle=\rho_{\rho_{1}} \kappa(M)$ for any machine $M$ and any stack-and-heap model $\left(S \times H, \circ^{\prime}, E\right)$.

Proof. As in Lemma 9.3, we show that $\left\langle s_{0}, e_{0}\right\rangle={ }_{\rho_{1}} \kappa(\gamma)$ for any instruction $\gamma$ in Definition 7.1. For any such $\gamma$, the formula $\kappa(\gamma)$ is of the form $(-A *-B)$ where $A$ and $B$ are of the form $l_{i}, l_{i} \vee l_{j}$ or $l_{i} * p_{k}$. Since $\left\langle s_{0}, e_{0}\right\rangle$ is a unit of the model and $\left\langle s_{0}, e_{0}\right\rangle \circ^{\prime}\langle s, h\rangle$ is undefined for $s \neq s_{0}$, we have

$$
\begin{aligned}
& \left\langle s_{0}, e_{0}\right\rangle \models_{\rho_{1}}-A *-B \\
\Leftrightarrow & \forall\langle s, h\rangle .\left\langle s_{0}, e_{0}\right\rangle \circ^{\prime}\langle s, h\rangle \text { defined and }\langle s, h\rangle \models_{\rho_{1}}-A \text { implies }\left\langle s_{0}, e_{0}\right\rangle \circ^{\prime}\langle s, h\rangle \models_{\rho_{1}}-B \\
\Leftrightarrow & \forall\left\langle s^{\prime}, h^{\prime}\right\rangle . s=s_{0} \text { and }\langle s, h\rangle \models_{\rho_{1}}-A \text { implies }\langle s, h\rangle \models-B \\
\Leftrightarrow & \forall h .\left\langle s_{0}, h\right\rangle \models_{\rho_{1}}-A \text { implies }\left\langle s_{0}, h\right\rangle \models_{\rho_{1}}-B .
\end{aligned}
$$

Recalling that $-A=A \rightarrow b$, and using Lemma 3.8, this amounts to showing, for any $h \in H$ :

$$
\llbracket A \rrbracket_{\rho_{1}} \cdot^{\prime}\left\{\left\langle s_{0}, h\right\rangle\right\} \subseteq \llbracket b \rrbracket_{\rho_{1}} \Rightarrow \llbracket B \rrbracket_{\rho_{1}} \cdot\left\{\left\langle s_{0}, h\right\rangle\right\} \subseteq \llbracket b \rrbracket_{\rho_{1}} .
$$

Assume $\llbracket A \rrbracket_{\rho_{1}} \cdot{ }^{\prime}\left\{\left\langle s_{0}, h\right\rangle\right\} \subseteq \llbracket b \rrbracket_{\rho_{1}}$, and let $d=\operatorname{domain}(h)$. By Lemma 9.8, we have $\left\langle s_{0}, h\right\rangle \in[d]$, and $\llbracket A \rrbracket_{\rho_{0}} \cdot\{\bar{d}\} \subseteq \llbracket b \rrbracket_{\rho_{0}}$. By Lemma 9.3, we have $e_{0} \models_{\rho_{0}} \kappa(\gamma)$ and thus (by the same reasoning as above) $\llbracket-A \rrbracket_{\rho_{0}} \subseteq \llbracket-B \rrbracket_{\rho_{0}}$, which means that $\llbracket A \rrbracket_{\rho_{0}} \cdot\{d\} \subseteq \llbracket b \rrbracket_{\rho_{0}}$ implies $\llbracket B \rrbracket_{\rho_{0}} \cdot\{d\} \subseteq \llbracket b \rrbracket_{\rho_{0}}$. Thus, we have $\llbracket B \rrbracket \rho_{\rho_{0}} \cdot\{d\} \subseteq \llbracket b \rrbracket_{\rho_{0}}$, whence Lemma 9.8 yields $\llbracket B \rrbracket_{\rho_{1}} \cdot\left\{\left\langle s_{0}, h\right\rangle\right\} \subseteq \llbracket \bar{b} \rrbracket_{\rho_{1}}$, as required.

LEMMA 9.10. $\left\langle s_{0}, e_{0}\right\rangle \models_{\rho_{1}} \mathrm{I} \wedge-l_{0}$ for any machine $M$ and any stack-and-heap model $\left(S \times H, \circ^{\prime}, E\right)$.

Proof. We have $\left\langle s_{0}, e_{0}\right\rangle \models_{\rho_{1}}$ I because $\left\langle s_{0}, e_{0}\right\rangle \in E$. By Lemma 9.4, we have $e_{0}=_{\rho_{0}}-l_{0}$ and thus $\rho_{0}\left(l_{0}\right) \subseteq \rho_{0}(b)$. Thus, we have

$$
\rho_{1}\left(l_{0}\right)=\bigcup_{d \in \rho_{0}\left(l_{0}\right)}[d] \subseteq \bigcup_{d \in \rho_{0}(b)}[d]=\rho_{1}(b),
$$

which implies that $\left\langle s_{0}, e_{0}\right\rangle \models \rho_{1}-l_{0}$, as required. 
Theorem 9.11. For any machine $M$ and configuration $\left\langle L_{i}, n_{1}, n_{2}\right\rangle$, if a sequent $\mathcal{F}_{M,\left\langle L_{i}, n_{1}, n_{2}\right\rangle}$ of the form

$$
\kappa(M) * l_{i} * p_{1}^{n_{1}} * p_{2}^{n_{2}} *\left(\mathrm{I} \wedge-l_{0}\right) \vdash b
$$

is valid in some concrete model listed in Section 2 then $\left\langle L_{i}, n_{1}, n_{2}\right\rangle \Downarrow_{M}$.

Proof. The case of Petri net marking models (Section 2.7) and integer partition models (Section 2.6) with their total $\circ$ can be covered by the original valuation $\rho_{0}$ from Definition 9.1. See also Section 9.5.

For other models, by taking appropriate locations $L$, record values $R V$, set of stacks $S$ and permission algebra $P$ (where $L$ is infinite), we may assume that $\mathcal{F}_{M,\left\langle L_{i}, n_{1}, n_{2}\right\rangle}$ is valid in a stack-and-heap model $\left(S \times H, \circ^{\prime}, E\right)$ as given in Definition 9.6. Thus, we have by definition of validity, and using Lemma 3.8:

$$
\begin{aligned}
\llbracket \kappa(M) * l_{i} * p_{1}^{n_{1}} * p_{2}^{n_{2}} *\left(\mathrm{I} \wedge-l_{0}\right) \rrbracket_{\rho_{1}} & \subseteq \llbracket b \rrbracket_{\rho_{1}} \\
\text { that is, } \llbracket \kappa(M) \rrbracket_{\rho_{1}} \cdot \llbracket l_{i} * p_{1}^{n_{1}} * p_{2}^{n_{2}} \rrbracket_{\rho_{1}} \cdot \llbracket \mathrm{I} \wedge-l_{0} \rrbracket_{\rho_{1}} & \subseteq \rho_{1}(b) .
\end{aligned}
$$

Taking into account that $\left\langle s_{0}, e_{0}\right\rangle \in \llbracket \kappa(M) \rrbracket_{\rho_{1}}$ and $\left\langle s_{0}, e_{0}\right\rangle \in \llbracket \mathrm{I} \wedge-l_{0} \rrbracket_{\rho_{1}}$ according to Lemmas 9.9 and 9.10 , respectively, we get:

$$
\llbracket l_{i} * p_{1}^{n_{1}} * p_{2}^{n_{2}} \rrbracket_{\rho_{1}} \subseteq \rho_{1}(b) .
$$

Using Lemma 9.7 and our definition of $\rho_{1}(b)$, we obtain:

$$
\bigcup_{d \in \llbracket l_{i} * p_{1}^{n_{1}} * p_{2}^{n_{2}} \rrbracket_{\rho_{0}}}[d] \subseteq \bigcup_{d \in \rho_{0}(b)}[d] .
$$

According to Lemma 9.2, each element from the set $\llbracket l_{i} * p_{1}^{n_{1}} * p_{2}^{n_{2}} \rrbracket_{\rho_{0}}$ uniquely determines the configuration $\left\langle L_{i}, n_{1}, n_{2}\right\rangle$, so that our construction of $\rho_{0}(b)$ yields $\left\langle L_{i}, n_{1}, n_{2}\right\rangle \Downarrow_{M}$.

\subsection{Valuation for the Total Multiset Model over a Fixed Finite Set $L$}

By the same token, we can prove that the following simplified valuation $\rho_{\text {mult }}$ provides the desired undecidability results for the total multiset model of Section 2.6 even for the finite multisets over a fixed finite set $L$. That is, we have $\left\langle L_{i}, n_{1}, n_{2}\right\rangle \Downarrow_{M}$ whenever the following sequent $\mathcal{F}_{M,\left\langle L_{i}, n_{1}, n_{2}\right\rangle}$ is valid in this particular total model:

$$
\kappa(M) * l_{i} * p_{1}^{n_{1}} * p_{2}^{n_{2}} *\left(\mathrm{I} \wedge-l_{0}\right) \vdash b .
$$

Definition 9.12. Given a machine $M$, we introduce the following valuation $\rho_{\text {mult }}$ for the finite multisets over a fixed finite set $L$ of Section 2.6:

$$
\begin{aligned}
\rho_{\text {mult }}\left(p_{1}\right) & =\{\{2\}\} \\
\rho_{\text {mult }}\left(p_{2}\right) & =\{\{3\}\} \\
\rho_{\text {mult }}\left(l_{i}\right) & =\{\{2 i+9\}\},
\end{aligned}
$$

where $i \geq-2$, and

$$
\rho_{\text {mult }}(b)=\bigcup_{\left\langle L_{i}, n_{1}, n_{2}\right\rangle \Downarrow_{M}} \llbracket l_{i} * p_{1}^{n_{1}} * p_{2}^{n_{2}} \rrbracket_{\rho_{\text {mult }}},
$$

where $b$ is the distinguished propositional variable introduced in Definition 7.3. (The reason behind our choice for $\rho_{\text {mult }}(b)$ is given in Section 7.1.)

In fact, the cardinality of the finite $L$ is proportional to the number of states of a universal Minsky machine. 
According to Section 9.2, for our practical heap-like models with their peculiar "disjoint heap composition", the corresponding $L$ is necessarily infinite, in order to guarantee that the valuations of the "counter propositions" $p_{1}$ and $p_{2}$ have infinitely many disjoint elements.

\section{FINITE APPROXIMATIONS IN INFINITE MODELS}

At first sight, our undecidability results for propositional separation logic seem to be at odds with the decidability of the quantifier-free fragment of a certain separation theory over an infinite heap model, due to Calcagno et al. [2001].

However, the crucial difference between our setting and theirs is that their decidability result is restricted to specific finite valuations $\rho$ such that $\rho(p)$ is finite for every atomic proposition $p$. More precisely, in Calcagno et al. [2001] each $p$ represents one cell, that is, a heap whose domain is a singleton. Their decidability result is nevertheless highly non-trivial because their language contains $*$ and the underlying separation model employs a nontotal $\circ$, so that, for example, whenever $\llbracket A \rrbracket_{\rho}$ is finite, $\llbracket A * B \rrbracket_{\rho}$ becomes infinite. Here, we investigate this phenomenon.

LEMMA 10.1. Let $\left(H, \circ,\left\{e_{0}\right\}\right)$ be a heap model from Section 2.1. There is an algorithm that, for any finite valuation $\rho$, decides whether $e_{0} \models_{\rho} \kappa(M)$ holds or not.

PRoof. In principle, this result can be deduced from Calcagno et al. [2001]. The following direct construction illustrates subtleties of the problem, caused by non-totality of the composition $\circ$.

As in Lemmas 9.3 and 9.9, we have to check whether

$$
e_{0} \models_{\rho} \kappa(\gamma)
$$

for any machine instruction $\gamma$ taken from Definition 7.1. For any such $\gamma$, the formula $\kappa(\gamma)$ is of the form $(-A *-B)$ where $A$ and $B$ are each of the form $l_{i},\left(l_{i} \vee l_{j}\right)$ or $\left(l_{i} * p_{k}\right)$. Note that, as $\rho$ is a finite valuation, $\llbracket A \rrbracket_{\rho}$ and $\llbracket B \rrbracket_{\rho}$ are also finite.

Checking whether $e_{0} \models_{\rho} \kappa(\gamma)$ thus means checking whether the following sentence is true or not (cf. (19)):

$$
\text { for all } h \in H, \llbracket A \rrbracket_{\rho} \cdot\{h\} \subseteq \rho(b) \text { implies } \llbracket B \rrbracket_{\rho} \cdot\{h\} \subseteq \rho(b) .
$$

By negating this statement, it suffices to check whether or not there is an $h \in H$ satisfying the following sentence:

$$
\llbracket A \rrbracket_{\rho} \cdot\{h\} \subseteq \rho(b) \text { and } \llbracket B \rrbracket_{\rho} \cdot\{h\} \nsubseteq \rho(b) .
$$

The tricky point, caused by the fact that $\circ$ is not total, is that the Eq. (32) may have an infinite number of solutions $h$. Nevertheless, we can construct, in advance, two finite sets $\mathcal{H}_{1}, \mathcal{H}_{2} \subseteq H$ such that, if Eq. (32) has a solution, then it has a solution $\widetilde{h}$ belonging to the finite set $\mathcal{H}_{1} \cup \mathcal{H}_{2}$.

In other words, statement (31), in which the range of the quantified $h$ is infinite, is equivalent to the following decidable statement, in which the finite range $\mathcal{H}_{1} \cup \mathcal{H}_{2}$ of the quantified $h$ can be computed in advance:

$$
\text { for all } h \in \mathcal{H}_{1} \cup \mathcal{H}_{2}, \llbracket A \rrbracket_{\rho} \cdot\{h\} \subseteq \rho(b) \text { implies } \llbracket B \rrbracket_{\rho} \cdot\{h\} \subseteq \rho(b) .
$$

The practical upshot of this transformation is that checking whether $e_{0} \models{ }_{\rho} \kappa(\gamma)$ can be done in a finite number of steps. It just remains to show how to construct the finite sets $\mathcal{H}_{1}$ and $\mathcal{H}_{2}$.

Let $\llbracket A \rrbracket_{\rho}=\left\{f_{1}, f_{2}, \ldots, f_{m}\right\}$ and $\llbracket B \rrbracket_{\rho}=\left\{g_{1}, \ldots, g_{t}\right\}$. Given a solution $h$ to (32), we consider two cases depending on whether or not $\llbracket A \rrbracket_{\rho} \cdot\{h\}=\emptyset$. 
C Case $\llbracket A \rrbracket_{\rho} \cdot\{h\} \neq \emptyset$. In this case, the product $f_{i} \circ h$ must be defined for some $i$, and, by (32), we have $f_{i} \circ h \in \rho(b)$. Thus, $h$ is guaranteed to belong to the finite set $\mathcal{H}_{1}$ of all "sub-heaps" of $\rho(b)$, defined as follows:

$$
\mathcal{H}_{1}=\left\{h \in H \mid \exists h^{\prime} \in H . h^{\prime} \circ h \in \rho(b)\right\} .
$$

-Case $\llbracket A \rrbracket_{\rho} \cdot\{h\}=\emptyset$. In this case, for each $i$ such that $1 \leq i \leq m$, the product $f_{i} \circ h$ is undefined and hence there is an $\ell_{i}$ such that $\ell_{i} \in \operatorname{domain}\left(f_{i}\right) \cap \operatorname{domain}(h)$. Notice that, by (32), we have $\llbracket B \rrbracket_{\rho} \cdot\{h\} \nsubseteq \rho(b)$. Consequently, for some $1 \leq j \leq t$, we have

$$
\operatorname{domain}\left(g_{j}\right) \cap\left\{\ell_{1}, \ell_{2}, \ldots, \ell_{m}\right\}=\emptyset .
$$

(Otherwise, for all $j$ we have that $\operatorname{domain}\left(g_{j}\right) \cap\left\{\ell_{1}, \ell_{2}, \ldots, \ell_{m}\right\} \neq \emptyset$, and thereby domain $\left(g_{j}\right) \cap$ domain $(h) \neq \emptyset$, resulting in $\llbracket B \rrbracket_{\rho} \cdot\{h\}=\emptyset$, which contradicts (32). )

Now we construct a new solution $\widetilde{h}$ in the following way. Let $\widetilde{a}$ be a fresh element of $L$ such that $\widetilde{a}$ does not occur in $\llbracket A \rrbracket_{\rho}, \llbracket B \rrbracket_{\rho}$ or $\rho(b)$, and let $\widetilde{b}$ be a fixed element of $R V$. Then, the domain of $\widetilde{h}$ is defined as the extended set $\left\{\ell_{1}, \ell_{2}, \ldots, \ell_{m}, \widetilde{a}\right\}$, and for each $x$ from domain $(\widetilde{h})$ we set:

$$
\widetilde{h}(x)=\widetilde{b} .
$$

It remains to show that $\widetilde{h}$ indeed satisfies the sentence (32).

First, for all $1 \leq i \leq m$, we have domain $\left(f_{i}\right) \cap \operatorname{domain}(\widetilde{h}) \neq \emptyset$ because $\ell_{i} \in \operatorname{domain}(\widetilde{h})$ by construction. This implies that $\llbracket A \rrbracket_{\rho} \cdot\{\widetilde{h}\}=\emptyset$.

Second, by (34), domain $\left(g_{j}\right)$ and domain $(\widetilde{h})$ are disjoint for some $1 \leq j \leq t$, so that $g_{j} \circ \widetilde{h}$ is defined and, because of the choice of $\widetilde{a}$, we have $g_{j} \circ \widetilde{h} \notin \rho(b)$, so $\llbracket B \rrbracket_{\rho} \cdot\{\widetilde{h}\} \nsubseteq \rho(b)$. Therefore, we can conclude that $\widetilde{h}$ is still a solution to (32).

Now we define a set $\mathcal{H}_{2}$ of "solution candidates" as follows. For each choice of $\ell_{1}$, $\ell_{2}, \ldots, \ell_{m}$ from domain $\left(f_{1}\right)$, domain $\left(f_{2}\right), \ldots$, domain $\left(f_{m}\right)$, respectively, $\mathcal{H}_{2}$ contains the heap $h^{\prime}$ defined as follows:

$$
\operatorname{domain}\left(h^{\prime}\right)=\left\{\ell_{1}, \ell_{2}, \ldots, \ell_{m}, \widetilde{a}\right\} \text { and } \forall x \in \operatorname{domain}\left(h^{\prime}\right) . h^{\prime}(x)=\widetilde{b} .
$$

It is clear that $\mathcal{H}_{2}$ is finite and contains every solution of the form $\widetilde{h}$, as required. This completes the proof.

Theorem 10.2. Let $\left(H, \circ,\left\{e_{0}\right\}\right)$ be a heap model from Section 2.1. Then, there is an algorithm that, for any finite valuation $\rho$, and any sequent $\mathcal{F}_{M,\left\langle L_{i}, n_{1}, n_{2}\right\rangle}$ of the form:

$$
\kappa(M) * l_{i} * p_{1}^{n_{1}} * p_{2}^{n_{2}} *\left(\mathrm{I} \wedge-l_{0}\right) \vdash b .
$$

decides whether this sequent is valid under the valuation $\rho$.

Proof. Given an $\mathcal{F}_{M,\left\langle L_{i}, n_{1}, n_{2}\right\rangle}$, we first use Lemma 10.1 to compute $\llbracket \kappa(M) \rrbracket_{\rho}$ and $\llbracket \mathrm{I} \wedge-l_{0} \rrbracket_{\rho}$ (note that each of these is either empty, or the singleton set $\left\{e_{0}\right\}$ ). If either of these sets is empty, then trivially $\mathcal{F}_{M,\left\langle L_{i}, n_{1}, n_{2}\right\rangle}$ is valid under the valuation $\rho$.

Otherwise, $\llbracket \kappa(M) \rrbracket_{\rho}=\llbracket \mathrm{I} \wedge-l_{0} \rrbracket_{\rho}=\left\{e_{0}\right\}$, so deciding the validity of $\mathcal{F}_{M,\left\langle L_{i}, n_{1}, n_{2}\right\rangle}$ under $\rho$ means deciding the inclusion

$$
\begin{aligned}
& \llbracket \kappa(M) * l_{i} * p_{1}^{n_{1}} * p_{2}^{n_{2}} *\left(\mathrm{I} \wedge-l_{0}\right) \rrbracket_{\rho} \subseteq \rho(b) \\
& \text { that is, } \llbracket \kappa(M) \rrbracket_{\rho} \cdot \llbracket l_{i} * p_{1}^{n_{1}} * p_{2}^{n_{2}} \rrbracket_{\rho} \cdot \llbracket\left(\mathrm{I} \wedge-l_{0}\right) \rrbracket_{\rho} \subseteq \rho(b) \\
& \text { that is, } \quad\left\{e_{0}\right\} \cdot \llbracket l_{i} * p_{1}^{n_{1}} * p_{2}^{n_{2}} \rrbracket_{\rho} \cdot\left\{e_{0}\right\} \subseteq \rho(b) \\
& \text { that is, } \quad \llbracket l_{i} * p_{1}^{n_{1}} * p_{2}^{n_{2}} \rrbracket_{\rho} \subseteq \rho(b)
\end{aligned}
$$

which is straightforward since both $\llbracket l_{i} * p_{1}^{n_{1}} * p_{2}^{n_{2}} \rrbracket_{\rho}$ and $\rho(b)$ are finite. 


\subsection{Noncompactness}

Corollary 10.3. We can construct a sequent $\mathcal{F}_{M,\left\langle L_{1}, n_{0}, 0\right\rangle}$ of the form

$$
\kappa(M) * l_{1} * p_{1}^{n_{0}} *\left(\mathrm{I} \wedge-l_{0}\right) \vdash b
$$

so that, for each heap model $\left(H, \circ,\left\{e_{0}\right\}\right)$ from Section 2.1,

(a) $\mathcal{F}_{M,\left\langle L_{1}, n_{0}, 0\right\rangle}$ is not valid in this model (and hence the corresponding computation of $M$ from $\left\langle L_{1}, n_{0}, 0\right\rangle$ does not terminate),

(b) but $\mathcal{F}_{M,\left\langle L_{1}, n_{0}, 0\right\rangle}$ is valid in this model under all finite valuations $\rho$.

Proof. Take $M$ such that $K_{M}=_{\text {def }}\left\{n \mid\left\langle L_{1}, n, 0\right\rangle \Downarrow_{M}\right\}$ is undecidable (the existence of such machines is guaranteed by Minsky [1967]). $K_{M}$ is recursively enumerable.

Now let $\left(H, \circ,\left\{e_{0}\right\}\right)$ be a heap model from Section 2.1. According to Figure 1 in Section 5, we have

$$
\begin{aligned}
K_{M} & =\left\{n \mid \mathcal{F}_{M,\left\langle L_{1}, n, 0\right\rangle} \text { provable in Minimal BBI }\right\} \\
& =\left\{n \mid \mathcal{F}_{M,\left\langle L_{1}, n, 0\right\rangle} \text { valid in all separation models }\right\} \\
& =\left\{n \mid \mathcal{F}_{M,\left\langle L_{1}, n, 0\right\rangle} \text { valid in }\left(H, \circ,\left\{e_{0}\right\}\right)\right\} .
\end{aligned}
$$

Next, we define

$$
W_{M}=_{\text {def }}\left\{n \mid \mathcal{F}_{M,\left\langle L_{1}, n, 0\right\rangle} \text { is not valid in }\left(H, \circ,\left\{e_{0}\right\}\right) \text { under some finite valuation } \rho\right\} .
$$

By Theorem 10.2, $W_{M}$ is also recursively enumerable.

However, by construction, $K_{M}$ and $W_{M}$ are disjoint. Moreover, since $K_{M}$ is recursively enumerable but undecidable, $W_{M}$ is not the whole complement of $K_{M}$. Therefore, we can find a number $n_{0}$ such that $n_{0} \notin K_{M} \cup W_{M}$. We can now complete the two parts of the corollary as follows:

(a) Since $n_{0} \notin K_{M}$, Theorem 9.11 implies that $\mathcal{F}_{M,\left\langle L_{1}, n_{0}, 0\right\rangle}$ is not valid in $\left(H, \circ,\left\{e_{0}\right\}\right)$.

(b) However, $n_{0} \notin W_{M}$ implies that $\mathcal{F}_{M,\left\langle L_{1}, n_{0}, 0\right\rangle}$ is valid in $\left(H, \circ,\left\{e_{0}\right\}\right)$ under all finite valuations $\rho$.

\section{EXTENSION TO CLASSICAL BI}

In this section, we extend our undecidability results to the class of "dualising separation models", whose proof-theoretical basis is given by the bunched logic Classical BI.

Definition 11.1. A CBI-model is given by $\left(H, \circ, e, \cdot^{-1}\right)$, where $\langle H, \circ,\{e\}\rangle$ is a separation model (with a single unit $e$ ) and ${ }^{-1}: H \rightarrow H$ satisfies $h \circ h^{-1}=e^{-1}$ for all $h \in H$.

The CBI-models we consider here form a subclass of the more general relational CBI-models given in Brotherston and Calcagno [2010].

Example 11.2. Examples of CBI-Models (cf. Brotherston and Calcagno [2010]).

(a) $\left([0,1], \circ, 0, \cdot^{-1}\right)$, where $x_{1} \circ x_{2}$ is $x_{1}+x_{2}$ but undefined when $x_{1}+x_{2}>1$. The inverse $x^{-1}$ is $1-x$.

(b) $(\Sigma, \circ, \varepsilon,-)$ where $\Sigma$ is any class of languages containing the empty language $\varepsilon$ and closed under union $\cup$ and complement - . Here $d_{1} \circ d_{2}$ is the union of disjoint languages $d_{1}$ and $d_{2}$ (in the overlapping case, $d_{1} \circ d_{2}$ is undefined). For example, $\Sigma$ may be the class of regular languages, or the class of finite and co-finite sets.

(c) Effect algebras [Foulis and Bennett 1994], which arise in the foundations of quantum mechanics, are exactly CBI-models with indivisible units.

(d) Permission algebras $(P, \bullet, \mathbb{1})$ [Bornat et al. 2005] enriched with a "formal unit" $e$ and "formal equalities" $e \bullet h=h \bullet e=e$ can be shown to be exactly nondegenerate (i.e., having more than one element) CBI-models with indivisible units. 
Definition 11.3. Following Definition 4.1, we introduce a second chain of logics as follows:

$$
\mathrm{BBI} \subseteq \mathrm{CBI} \subseteq \mathrm{CBI}+\mathrm{eW} \subseteq \mathrm{CBI}+\mathrm{W} .
$$

-Classical BI, a.k.a. CBI Brotherston and Calcagno [2010] is obtained from BBI by extending its language with a constant $\widetilde{\mathrm{I}}$, and adding the axiom $\sim A \vdash A$, where $\sim A$ is an abbreviation for $(A \rightarrow \widetilde{\mathrm{I}})$.

$-\mathrm{CBI}+\mathrm{eW}$ is obtained by extending $\mathrm{CBI}$ with the restricted $*$-weakening $\mathrm{I} \wedge(A * B) \vdash A$.

$-\mathrm{CBI}+\mathrm{W}$ is obtained by extending $\mathrm{CBI}$ with the unrestricted $*$-weakening $A * B \vdash A$.

Validity of CBI-formulas with respect to CBI-models $\left(H, \circ, e, .^{-1}\right)$ is given by extending the satisfaction relation in Definition 3.9 with the clause:

$$
h \models_{\rho} \widetilde{\mathrm{I}} \Leftrightarrow h \neq e^{-1} .
$$

Proposition 11.4. If $A$ is provable in $\mathrm{CBI}$, then $A$ is valid in all CBI-models, and if $A$ is provable in $\mathrm{CBI}+\mathrm{eW}$, then $A$ is valid in all $\mathrm{CBI}-$ models with indivisible units.

Proof. Since CBI-models are special cases of separation models, and BBI is sound wrt. separation models (Proposition 4.2), it suffices to show for the first part that the CBI axiom $\sim A \vdash A$ is valid in an arbitrary $\operatorname{CBI}-$ model $\left(H, \circ, e, \cdot^{-1}\right)$.

First, we observe that for any $h \in H$, the element $h^{-1}$ is the unique element such that $h \circ h^{-1}=e^{-1}$. For suppose that $h \circ x=e^{-1}$, then we have by associativity and commutativity of $\circ$ :

$$
e^{-1} \circ x=\left(h \circ h^{-1}\right) \circ x=(h \circ x) \circ h^{-1}=e^{-1} \circ h^{-1},
$$

whence we obtain $x=h^{-1}$ by cancellativity ${ }^{3}$ of $\circ$. As an immediate corollary, we also have $\left(h^{-1}\right)^{-1}=h$.

Now by the definition of validity and recalling that $\sim A=_{\text {def }}(A * \widetilde{\mathrm{I}})$, we have:

$$
h \models_{\rho} \sim A \Leftrightarrow \forall h^{\prime} . h \circ h^{\prime} \text { defined and } h^{\prime} \models_{\rho} A \text { implies } h \circ h^{\prime} \neq e^{-1} .
$$

Since $h^{-1}$ is the unique element of $H$ such that $h \circ h^{-1}=e^{-1}$, we obtain:

$$
h \models_{\rho} \sim A \Leftrightarrow h^{-1} \nvdash_{\rho} A .
$$

Thus, using the fact that $\left(h^{-1}\right)^{-1}=h$, we have:

$$
h=_{\rho} \sim \sim A \Leftrightarrow h^{-1} \not \models_{\rho} \sim A \Leftrightarrow\left(h^{-1}\right)^{-1} \models_{\rho} A \Leftrightarrow h \models_{\rho} A
$$

and so $\sim \sim \vdash A$ (as well as its converse) is valid as required.

For soundness of $\mathrm{CBI}+\mathrm{eW}$, the fact that restricted $*$-weakening holds in all CBImodels with indivisible units follows from the fact that it holds in all separation models with indivisible units, as established in Proposition 4.2.

COROLLARY 11.5. Interpreting $\subset$ as strict inclusion between the set of sequents provable in each system using only the language of BBI, we have:

$$
\mathrm{BBI} \subset \mathrm{CBI} \subset \mathrm{CBI}+\mathrm{eW} \subset \mathrm{CBI}+\mathrm{W} .
$$

Proof. The nonstrict versions of the inclusions hold easily by construction. The noninclusion CBI $\nsubseteq$ BBI was established in Brotherston and Calcagno [2010]. By the

\footnotetext{
${ }^{3}$ The original definition of a CBI-model in Brotherston and Calcagno [2010] requires as an axiom that the dual $h^{-1}$ be unique for each $h$, whereas here we drop this requirement in favour of cancellativity of $\circ$, which is arguably more natural.
} 
same arguments as in Theorem 3.10 and Corollary 4.4, restricted $*$-weakening is not valid in all CBI-models, and unrestricted $*$-weakening is not valid in all CBI-models with indivisible units. Thus, the noninclusions $\mathrm{CBI}+\mathrm{W} \nsubseteq \mathrm{CBI}+\mathrm{eW} \nsubseteq \mathrm{CBI}$ hold by Proposition 11.4.

Proposition 11.6. $\mathrm{CBI}+\mathrm{W}$ is ordinary classical logic.

Proof. By the same argument as in Proposition 4.7, the presence of unrestricted *-weakening forces the equivalences $A * B \equiv A \wedge B, A * B \equiv A \rightarrow B$ and $\mathrm{I} \equiv \mathrm{\top}$.

It just remains to show that $\widetilde{\mathrm{I}} \equiv \perp$. To see this, first note that $\perp \vdash \widetilde{\mathrm{I}}$ is trivially provable by the usual ex falso quodlibet of classical logic. For the reverse direction, first note that $(\perp * \widetilde{\mathrm{I}}) \vdash \top$ is trivially provable. As a consequence, $(\top * \widetilde{\mathrm{I}}) \vdash((\perp * \widetilde{\mathrm{I}}) * \widetilde{\mathrm{I}})$ is also provable. By the CBI axiom, we have $((\perp * \widetilde{\mathrm{I}}) * \widetilde{\mathrm{I}}) \vdash \perp$, whence by transitivity of $\vdash$ we obtain $(T * \widetilde{\mathrm{I}}) \vdash \perp$. By the equivalence $\mathrm{I} \equiv T$, we then have $(\mathrm{I} * \widetilde{\mathrm{I}}) \vdash \perp$. Since $\widetilde{\mathrm{I}} \vdash(\mathrm{I} * \widetilde{\mathrm{I}})$ is easily derivable, we have $\widetilde{\mathrm{I}} \vdash \perp$ provable by transitivity as required.

We observe that, as a consequence, $\sim A \equiv A \rightarrow \perp \equiv \neg A$.

Since (Minimal) BBI-provability implies CBI-provability, to establish undecidability for CBI, it suffices (see Figure 1) to prove the analogue of Theorem 9.5 for a CBI-model.

Definition 11.7. We introduce the $R A M$-codomain model $\left(\mathcal{D}^{+}, \circ, e_{0},{ }^{-1}\right)$, where $\mathcal{D}^{+}$is the class of finite and co-finite subsets of $\mathbb{N}$, $\circ$ is the union of disjoint sets (and undefined for nondisjoint sets), the unit $e_{0}$ is $\varnothing$ and $\cdot^{-1}$ is set complement.

By extending the valuation $\rho_{0}$ in Definition 9.1, we define a valuation $\rho_{C}$ for $\left(\mathcal{D}^{+}, \circ, e_{0},{ }^{-1}\right)$ as follows: $\rho_{C}$ coincides with $\rho_{0}$ on all atomic propositions except $b$, and

$$
\rho_{C}(b)=\rho_{0}(b) \cup\left\{d \in \mathcal{D}^{+} \mid d \text { is co-finite }\right\} .
$$

\section{Lemma 11.8. $e_{0} \models_{\rho_{C}} \kappa(M)$ for any machine $M$.}

Proof. As in Lemma 9.3, we must show $e_{0}=_{\rho_{C}} \kappa(\gamma)$ for any instruction $\gamma$. Here we only examine the case of an increment instruction $\gamma=\left(L_{i}: c_{k}++\right.$; goto $L_{j}$;) for $k=1$. We note that we have, as in Lemma 9.3,

$$
d \models_{\rho_{C}}-A \Leftrightarrow \forall d^{\prime} . d, d^{\prime} \text { disjoint and } d^{\prime} \models_{\rho_{C}} A \text { implies } d \circ d^{\prime} \in \rho_{C}(b) .
$$

We have $\kappa(\gamma)=\left(-\left(l_{j} * p_{1}\right) *-l_{i}\right)$. To show $e_{0}==_{\rho_{C}} \kappa(\gamma)$, we must show for any $d \in \mathcal{D}^{+}$, as in the corresponding case of Lemma 9.3,

$$
d \models_{\rho_{C}}-\left(l_{j} * p_{1}\right) \text { implies } d \models_{\rho_{C}}-l_{i} .
$$

Assuming that $d \models_{\rho_{C}}-\left(l_{j} * p_{1}\right)$, there are two cases to consider.

First, if $d$ is finite then we can find (as in the corresponding case of Lemma 9.3) $d_{j} \in \rho_{C}\left(l_{j}\right)$ and $d_{1} \in \rho_{C}\left(p_{1}\right)$ such that $d, d_{j}$ and $d_{1}$ are disjoint, and thus in particular $d_{j} \circ d_{1} \models_{\rho_{C}} l_{j} * p_{1}$. Using equivalence (35), we have:

$$
\begin{aligned}
d \circ d_{j} \circ d_{1} & \in \rho_{C}(b) \\
& =\rho_{0}(b) \cup\left\{d \in \mathcal{D}^{+} \mid d \text { is co-finite }\right\} .
\end{aligned}
$$

Since $d, d_{j}$, and $d_{1}$ are finite, we must have $d \circ d_{j} \circ d_{1} \in \rho_{0}(b)$ and thus, by the same argument as in the corresponding case of Lemma 9.3, $d \in \llbracket p_{1}^{n_{1}} * p_{2}^{n_{2}} \rrbracket \rho_{\rho_{0}}$ where $\left\langle L_{j}, n_{1}+\right.$ $\left.1, n_{2}\right\rangle \Downarrow_{M}$ and thus $\left\langle L_{i}, n_{1}, n_{2}\right\rangle \Downarrow_{M}$ by applying the instruction $\gamma$. Consequently, $d \circ d^{\prime} \in$ $\rho_{0}(b) \subseteq \rho_{C}(b)$ for any $d^{\prime}$ such that $d, d^{\prime}$ are disjoint and $d^{\prime} \in \rho_{C}\left(l_{i}\right)$. Thus, by (35), we have $\bar{d}=_{\rho_{C}}-l_{i}$ as required.

If, on the other hand, $d$ is co-finite then, for any choice of $d^{\prime} \in \rho_{C}\left(l_{i}\right)$ with $d, d^{\prime}$ disjoint, $d \circ d^{\prime}$ must also be co-finite, in which case $d \circ d^{\prime} \in \rho_{C}(b)$ because $\rho_{C}(b)$ contains all co-finite sets. Thus, again by (35), we have $d \models_{\rho_{C}}-l_{i}$ as required.

The other cases follow from their analogues in Lemma 9.3 in a similar fashion. 
LEMMA 11.9. $e_{0} \models_{\rho_{C}} \mathrm{I} \wedge-l_{0}$ for any machine $M$.

Proof. Similar to Lemma 9.4.

THEOREM 11.10. If the sequent $\kappa(M) * l_{i} * p_{1}^{n_{1}} * p_{2}^{n_{2}} *\left(\mathrm{I} \wedge-l_{0}\right) \vdash b$ is valid in the model $\left(\mathcal{D}^{+}, \circ, e_{0},{ }^{-1}\right)$, then $\left\langle L_{i}, n_{1}, n_{2}\right\rangle \Downarrow_{M}$.

Proof. By the definition of validity, and using Lemma 3.8, we have:

$$
\begin{aligned}
\llbracket \kappa(M) * l_{i} * p_{1}^{n_{1}} * p_{2}^{n_{2}} *\left(\mathrm{I} \wedge-l_{0}\right) \rrbracket_{\rho_{C}} & \subseteq \rho_{C}(b) \\
\text { that is, } \llbracket \kappa(M) \rrbracket_{\rho_{C}} \cdot \llbracket l_{i} * p_{1}^{n_{1}} * p_{2}^{n_{2}} \rrbracket_{\rho_{C}} \cdot \llbracket \mathrm{I} \wedge-l_{0} \rrbracket_{\rho_{C}} & \subseteq \rho_{C}(b) .
\end{aligned}
$$

By Lemmas 11.8 and 11.9, we have in particular:

$$
\llbracket l_{i} * p_{1}^{n_{1}} * p_{2}^{n_{2}} \rrbracket_{\rho_{C}} \subseteq \rho_{C}(b) .
$$

Since $\rho_{C}$ coincides with $\rho_{0}$ on all atomic propositions except $b$, we have $\llbracket l_{i} * p_{1}^{n_{1}} * p_{2}^{n_{2}} \rrbracket_{\rho_{C}}=$ $\llbracket l_{i} * p_{1}^{n_{1}} * p_{2}^{n_{2}} \rrbracket_{\rho_{0}}$, so that $\left\langle L_{i}, n_{1}, n_{2}\right\rangle$ is uniquely determined according to Lemma 9.2. In particular, $\llbracket l_{i} * p_{1}^{n_{1}} * p_{2}^{n_{2}} \rrbracket_{\rho_{C}}$ is finite, so our construction of $\rho_{C}(b)$ yields $\left\langle L_{i}, n_{1}, n_{2}\right\rangle \Downarrow_{M}$.

Again, based on Figure 1, we can assert the following:

CoROLlary 11.11. The following properties of formulas are undecidable, even when restricted to the language $(\wedge, \rightarrow, \mathrm{I}, *, \rightarrow)$ of Minimal $\mathrm{BBI}^{4}$ :

(a) provability in $\mathrm{CBI}$;

(b) provability in $\mathrm{CBI}+\mathrm{eW}$;

(c) validity in the class of all CBI-models;

(d) validity in the class of all CBI-models with indivisible units;

(e) validity in the RAM-codomain model $\left(\mathcal{D}^{+}, \circ, e_{0},{ }^{-1}\right)$.

Proof. Similar to Corollary 5.1.

Corollary 11.12. Neither CBI nor CBI+eW has the finite model property.

PROOF. Similar to Corollary 5.2.

\section{CONCLUDING REMARKS}

Our main contribution in this article is that heap-like memory models, of the type considered in Section 2, have a very complicated logical structure even at the propositional level.

(A) Separation logic, which provides us with an effective language for reasoning about such memory models, is undecidable even at the purely propositional level.

(B) Moreover, we have established that for any particular heap-like memory model drawn from the literature, validity in that model is undecidable even for purely propositional formulas in this language. Corollary 5.1(h) provides an infinite number of concrete undecidable models of practical and theoretical importance. That is, however we choose $L, R V, S$ and $P$ in Section 2 (with $L$ infinite and $R V, S, P$ possibly degenerate), we always get an undecidable model.

(C) We also have established the undecidability of validity in various classes of separation models, and of provability in BBI and CBI and their siblings.

\footnotetext{
${ }^{4}$ On the surface, it seems that we are within the framework of Corollary 5.1, since formulas are restricted to the language $(\wedge, \rightarrow, \mathrm{I}, *, *)$, which does not include the new CBI constant $\widetilde{\mathrm{I}}$. However, the problem remains nontrivial because of the fact that $\mathrm{CBI}$ is not a conservative extension of $\mathrm{BBI}$.
} 
(D) In fact, to obtain a new exhibit for the "Undecidability Zoo" - that is, a minimal version of BBI that is "as simple as possible" - we only need to add classical conjunction and implication to the multiplicatives, without invoking either classical negation or falsum (see Section 6).

(E) From a technical point of view, we kill all our "undecidable birds" with one "stone": a single direct encoding of Minsky machines. This single encoding suffices to cover all cases of interest (see Figure 1).

\subsection{New Principles for Separation Logic}

In proving our main undecidability results on separation logic, we have found a number of new principles which should be taken into account at the level of the multiplicative unit I.

(i) Though *-contraction, $A \vdash A * A$, is not generally valid in separation logic, in Lemma 6.4, we deduce the following restricted $*$-contraction

$$
\mathrm{I} \wedge A \vdash A * A \text {. }
$$

(ii) As for the systems $\mathrm{BBI}+\mathrm{eW}$ and $\mathrm{CBI}+\mathrm{eW}$ newly established in this article, we remark that the memory models of theoretical/practical importance employed in the literature (cf. Section 2) all have indivisible units in the sense of Definition 3.4, which is exactly axiomatized by the restricted $*$-weakening principle of $\mathrm{BBI}+\mathrm{eW}$ and CBI+eW (see Theorem 3.10):

$$
\mathrm{I} \wedge(A * B) \vdash A \text {. }
$$

This principle is in accordance with the following law on "conservation of matter":

"The empty memory cannot be split into nonempty pieces."

\subsection{Separation Logic vs. Linear Logic}

Our undecidability results also shed new light on the correlations between separation logic and linear logic.

From the point of view of logical principles, there are clear differences between the two. For example, distributivity of additive conjunction over disjunction:

$$
A \wedge(B \vee C) \equiv(A \wedge B) \vee(A \wedge C)
$$

holds even in BI but fails in linear logic.

More specific to Boolean BI, the restricted $*$-contraction:

$$
\mathrm{I} \wedge A \vdash A * A
$$

holds even in Minimal BBI as shown by our Lemma 6.4, but this too fails in linear logic.

Finally, while adding the unrestricted $*$-weakening $(A * B) \vdash A$ to linear logic gives us a rich structure of affine logic, adding it to BBI forces a collapse into classical logic (Proposition 4.7).

From a semantic perspective, the precise expression of properties of memory in separation logic is based on the fact that we have:

$$
\llbracket A * B \rrbracket_{\rho}=\llbracket A \rrbracket_{\rho} \cdot \llbracket B \rrbracket_{\rho},
$$

that is, the interpretation of $A * B$ is exactly the product of the interpretations of $A$ and $B$. (This fact is also of crucial importance to its undecidability.) Linear logic interpretations deal only with sets that are closed with respect to a certain closure operator $\mathrm{Cl}$, which, in particular, violates this exact equality. Indeed, the same is true of BI interpretations [O'Hearn and Pym 1999]. Not only is this less precise, it admits 
no possibility of finite valuations in these logics since, for example, in linear logic even $C l(\emptyset)$ is always infinite.

\subsection{Undecidability vs. Decidable Fragments of Memory Models}

Finally, our undecidability results for concrete heap-like models give new insights into the nature of decidable fragments of memory models such as those given in Berdine et al. [2004] and Calcagno et al. [2001], as well as imposing boundaries on decidability. For example, we can deduce that to obtain decidability in a heap-like model, one should either give up arbitrary infinite valuations (as in Calcagno et al. [2001]) or restrict the formula language (as in Berdine et al. [2004]).

\section{ACKNOWLEDGMENTS}

We are greatly indebted to Peter O'Hearn and Hongseok Yang for their fruitful discussions and suggestions. Thanks to Sam Staton for his observation on effect algebras [Foulis and Bennett 1994], and to Tadeusz Litak who has attracted our attention to Kurucz et al. [1995]. We owe special thanks to the referees for their scrupulous and insightful comments, which have allowed us to significantly improve the exposition.

\section{REFERENCES}

A. Ahmed, L. Jia, and D. Walker. 2003. Reasoning about hierarchical storage. In Proceedings of LICS-18. IEEE Computer Society, 33-44.

A. V. Aho, J. E. Hopcroft, and J. D. Ullman. 1974. The Design and Analysis of Computer Algorithms. AddisonWesley.

G. E. Andrews. 1976. The Theory of partitions. Encyclopedia of Mathematics and Its Applications. AddisonWesley.

N. P. Benton, G. M. Bierman, V. de Paiva, and M. Hyland. 1993. A term calculus for intuitionistic linear logic. In Proceedings of TLCA-1. Springer, 75-90.

J. Berdine, C. Calcagno, and P. O'Hearn. 2004. A decidable fragment of separation logic. In Proceedings of FSTTCS-24. Springer, 97-109.

R. Bornat, C. Calcagno, P. O'Hearn, and M. Parkinson. 2005. Permission accounting in separation logic. In Proceedings of POPL-32. 59-70.

J. Brotherston. 2012. Bunched logics displayed. Studia Logica 100, 6, 1223-1254.

J. Brotherston and C. Calcagno. 2010. Classical BI: Its semantics and proof theory. Logical Meth. Comput. Sci. 6, 3.

J. Brotherston and M. Kanovich. 2010. Undecidability of propositional separation logic and its neighbours. In Proceedings of LICS-25. IEEE Computer Society, 137-146.

J. Brotherston and J. Villard. 2014. Parametric completeness for separation theories. In Proceedings of POPL-41. ACM. 453-464.

C. Calcagno, D. Distefano, P. O’Hearn, and H. Yang. 2011. Compositional shape analysis by means of biabduction. J. ACM 58, 6.

C. Calcagno, P. O'Hearn, and H. Yang. 2007. Local action and abstract separation logic. In Proceedings of LICS-22. IEEE Computer Society, 366-378.

C. Calcagno, H. Yang, and P. W. O'Hearn. 2001. Computability and complexity results for a spatial assertion language for data structures. In Proceedings of FSTTCS-21. Springer, 108-119.

D. Distefano and M. Parkinson. 2008. jStar: Towards practical verification for Java. In Proceedings of OOPSLA-23. ACM, 213-226.

M. Dodds, X. Feng, M. Parkinson, and V. Vafeiadis. 2009. Deny-guarantee reasoning. In Proceedings of ESOP-18. Springer, 363-377.

D. Foulis and M. Bennett. 1994. Effect algebras and unsharp quantum logics. Found. Phys. 24, 1331-1352.

D. Galmiche and D. Larchey-Wendling. 2006. Expressivity properties of Boolean BI through relational models. In Proceedings of FSTTCS-26. Springer, 357-368.

D. Galmiche, D. Méry, and D. Pym. 2005. The semantics of BI and resource tableaux. Math. Struct. Comput. Sci. 15, 1033-1088.

P. Gardner, S. Maffeis, and G. D. Smith. 2012. Towards a program logic for JavaScript. In Proceedings of POPL-39. 31-44. 
J.-Y. Girard and Y. Lafont. 1987. Linear logic and lazy computation. In Proceedings of TAPSOFT'87. SpringerVerlag, 52-66.

A. Gotsman, B. Cook, M. Parkinson, and V. Vafeiadis. 2009. Proving that non-blocking algorithms don't block. In Proceedings of POPL-36. ACM, 16-28.

S. Ishtiaq and P. W. O'Hearn. 2001. BI as an assertion language for mutable data structures. In Proceedings of POPL-28. ACM, 14-26.

E. Jipsen. 1992. Computer aided investigations of relation algebras. Ph.D. dissertation, Vanderbilt University, Nashville, Tennessee.

M. Kanovich. 1992. Horn programming in linear logic is NP-complete. In Proceedings of LICS-7. IEEE Computer Society, 200-210.

M. Kanovich. 1995. The direct simulation of Minsky machines in linear logic. In Advances in Linear Logic, London Mathematical Society Lecture Notes Series, vol. 222, Cambridge University Press, 123-145.

A. Kurucz, I. Nemeti, I. Sain, and A. Simon. 1995. Decidable and undecidable modal logics with a binary modality. J. Logic, Lang. Inf. 4, 191-206.

A. Kurucz. 1997. Decision problems in algebraic logic. Ph.D. dissertation, Hungarian Academy of Sciences.

D. Larchey-Wendling and D. Galmiche. 2010. The undecidability of Boolean BI through phase semantics. In Proceedings of LICS-25. IEEE Computer Society, 140-149.

D. Larchey-Wendling and D. Galmiche. 2013. Nondeterministic phase semantics and the undecidability of Boolean BI. ACM Trans. Comput. Logic 14, 1, 6.

M. Minsky. 1967. Computation: Finite and Infinite Machines. Prentice-Hall, Inc.

T. Murata. 1989. Petri nets: Properties, analysis and applications. Proc. IEEE 77, 4, 541-580.

P. W. O'Hearn and D. J. Pym. 1999. The logic of bunched implications. Bull. Symb. Logic 5, 2, $215-244$.

M. Parkinson and G. Bierman. 2008. Separation logic, abstraction and inheritance. In Proceedings of POPL35. ACM, 75-86.

M. Parkinson, R. Bornat, and C. Calcagno. 2006. Variables as resource in Hoare logics. In Proceedings of LICS-21. IEEE Computer Society, 137-146.

J. L. Peterson. 1981. Petri Net Theory and the Modeling of Systems. Prentice-Hall.

D. Pym. 2002. The Semantics and Proof Theory of the Logic of Bunched Implications. Applied Logic Series. Kluwer.

D. Pym, P. O'Hearn, and H. Yang. 2004. Possible worlds and resources: The semantics of BI. Theoret. Comput. Sci. 315, 1, 257-305.

J. C. Reynolds. 2002. Separation logic: A logic for shared mutable data structures. In Proceedings of LICS-17. IEEE Computer Society, 55-74.

R. Statman. 1979. Intuitionistic propositional logic is polynomial-space complete. Theoret. Comput. Sci. 9, 67-72.

H. Yang, O. Lee, J. Berdine, C. Calcagno, B. Cook, D. Distefano, and P. O'Hearn. 2008. Scalable shape analysis for systems code. In Proceedings of CAV-20. Springer, 385-398.

Received April 2012; revised February 2013, August 2013; accepted November 2013 